\title{
Quantization of Chern-Simons Gauge Theory with Complex Gauge Group
}

\author{
Edward Witten ${ }^{\star}$ \\ School of Natural Sciences, Institute for Advanced Study, Olden Lane, Princeton, NJ 08540, USA \\ Received January 8, 1990
}

\begin{abstract}
The canonical quantization of Chern-Simons gauge theory in $2+1$ dimensions is generalized from the case in which the gauge group is a compact Lie group $G$ to the case in which the gauge group is a complex Lie group $G_{\mathbb{C}}$. Though the physical Hilbert spaces become infinite dimensional in the latter case, the quantization can be described as precisely as for compact gauge groups and using similar methods. The special case in which the gauge group is $S L(2, \mathbb{C})$ gives a description of $2+1$ dimensional quantum gravity with Lorentz signature and positive cosmological constant or with Euclidean signature and negative cosmological constant. While it is not clear whether there is a $1+1$ dimensional conformal field theory related to these $2+1$ dimensional models, there are natural, computable candidates for the central charge and the conformal blocks of such a hypothetical theory.
\end{abstract}

\section{Introduction}

In Yang-Mills theory, with a gauge group $G$, letting $A$ denote a connection on a $G$ bundle $E$ over a space-time manifold $M$, the conventional action functional is

$$
I=\frac{1}{4 g^{2}} \int_{M} \operatorname{Tr}\left(F_{i j} F^{i j}\right)
$$

Here "Tr" represents an invariant and non-degenerate quadratic form on the Lie algebra $\mathbf{g}$ of $G$, customarily denoted by $(a, b)=\operatorname{Tr}(a b)$, and $F=d A+A \wedge A$ is the Yang-Mills field strength.

In studying the theory associated with the Lagrangian (1.1), one usually requires that the quadratic form defined by "Tr" should be positive definite, but the reason for this requirement is not always stated accurately. If the quadratic form used in (1.1) is indefinite, then the kinetic energy of the gauge bosons is indefinite. It is

* Research supported in part by NSF Grant 86-20266 and NSF Waterman Grant 88-17521 
not the case that this leads to a non-unitary theory. In fact, canonical quantization of a bosonic theory with a real Lagrangian always gives a unitary theory, a fact which is evident at a very elementary level by noting that the canonical commutation relations

$$
[p, q]= \pm i
$$

are compatible with unitarity for either choice of sign; in fact, these commutation relations have the unitary representation $p= \pm i d / d q$. What is wrong with (1.1) if "Tr" is not positive definite is that the energy is unbounded below, a fact that is evident classically and persists in the quantum theory. (In two space-time dimensions, in which the quantum field theory defined by (1.1) is exactly soluble, it is amusing to check explicitly that the theory determined by this Lagrangian with a non-positive choice of "Tr" is unitary but has indefinite energy.)

It is interesting to note that for fermions the situation is reversed. The canonical commutation relations for hermitian fermi variables $\psi_{k}$,

$$
\left\{\psi_{k}, \psi_{l}\right\}=m_{k l},
$$

are compatible with unitarity if and only if the matrix $m$ is positive definite. On the other hand, for fermions there is no issue of positivity of the energy. The classical energy is always indefinite for fermions, and at the quantum level positivity of the energy is always achieved by filling the Dirac sea. Thus, for bosons, unitarity is automatic but positivity of the energy is a non-trivial requirement; for fermions, unitarity is a non-trivial requirement but positivity of the energy is automatic.

It is because one usually wishes theories with positive energy that Yang-Mills theory is usually studied only for certain types of gauge group (compact groups times factors of $\mathbb{R}$ ). However, for Chern-Simons gauge theory in $2+1$ dimensions (which is governed by a Lagrangian that we will write explicitly in the next section), the situation is completely different. Regardless of the choice of the gauge group $G$, this theory is unitary because bosonic theories with real Lagrangians are always unitary, and it has a Hamiltonian which is bounded below because in fact - by virtue of general covariance - the Hamiltonian in Chern-Simons gauge theory is zero. It is a natural problem to try to understand the unitary and generally covariant quantum field theories determined by Chern-Simons Lagrangians for gauge groups more general than those that have been treated hitherto.

One motivation for this investigation is that the $2+1$ dimensional ChernSimons theories with compact gauge group give natural explanations [1] for many constructions in conformal field theory and integrable lattice models that have been intensively studied in recent years. The $2+1$ dimensional theories with more general gauge groups are likely to give rise to significant generalizations of these constructions - generalizations that are not presently known and that may be very difficult to guess from a purely $1+1$ dimensional point of view.

A further reason for the interest of this problem is the light that it may shed on quantum gravity. In fact [2-4], $2+1$ dimensional general relativity (for spacetimes of Lorentz signature) is related to Chern-Simons gauge theory with gauge group $S L(2, \mathbb{C}), I S O(2,1)$, or $S L(2, \mathbb{R}) \times S L(2, \mathbb{R})$ depending on whether the cosmological constant is positive, zero, or negative. In each case, the possible invariant quadratic forms are indefinite, so these gauge groups would be forbidden in ordinary Yang-Mills theory. General relativity is of course a unitary theory (with 
zero energy on a compact spatial slice because of general covariance), or it would not have won physical acceptance. Its equivalence with Chern-Simons gauge theory is possible because the latter, even with non-compact gauge groups, is unitary, and has vanishing Hamiltonian, as we have just explained.

$2+1$ dimensional general relativity with zero cosmological constant corresponds to an $\operatorname{ISO}(2,1)$ theory that is soluble in a particularly explicit way [5]; some of the physical properties of this theory have been investigated recently [6]. When the cosmological constant is not zero, the understanding of $2+1$ dimensional general relativity is much more difficult. The purpose of the present paper is to understand in detail the canonical quantization in the case that the cosmological constant is positive, corresponding to $S L(2, \mathbb{C})$ gauge group. Actually, it turns out that (for the issues we will consider) there is no additional difficulty to consider the more general case in which the gauge group is the complexification $G_{\mathbb{C}}$ of an arbitrary compact Lie group $G$, and we will study this more general case.

The relation between $1+1$ dimensional gravity and gauge theory that has been claimed in [7] appears to be a more difficult counterpart of the relation that arises straightforwardly in $2+1$ dimensions. Even if one is mainly interested in $1+1$ dimensions, it is possible that the relation between gravity and gauge theory can be best understood by starting in $2+1$ dimensions. This thought was the motivation for some recent work [8] on $2+1$ dimensional $S L(2, \mathbb{R})$ Chern-Simons theory.

Need For $A 2+1$ Dimensional Viewpoint. The fact that the hypothetical $1+1$ dimensional counterparts of $2+1$ dimensional Chern-Simons theory with complex gauge group are not already understood adds to the fascination of the $2+1$ dimensional models, but makes it much more difficult to study them. Thus, in the case of the $2+1$ dimensional theory with compact group, it originally seemed natural [1] to use the $2+1$ dimensional theory to explain qualitative facts in $1+1$ dimensions, while using known results in $1+1$ dimensions to give quantitative results in $2+1$ dimensions. In further developments $[9,10]$, many aspects of the canonical quantization and the relation between $1+1$ and $2+1$ dimensions were illuminated more precisely, but quantitative understanding of the $2+1$ dimensional theory still rested at crucial points on known results in $1+1$ dimensions.

To understand a $2+1$ dimensional theory whose $1+1$ dimensional counterpart is not yet known, it is of course necessary to have a self-contained $2+1$ dimensional approach. Recently [11], the canonical quantization of the $2+1$ dimensional theory, with compact gauge group, has been developed in a self-contained fashion in $2+1$ dimensions, without need for reference to already known results in $1+1$ dimensions. Apart from giving purely $2+1$ dimensional derivations of the traditional $1+1$ dimensional results, such methods make it possible to study $2+1$ dimensional theory whose $1+1$ dimensional counterparts are not known. What will be done in this paper is precisely to apply the methods of [11] to the $2+1$ dimensional theory with complex gauge group. The $2+1$ dimensional problem with complex gauge group also has an alternative algebra-geometric treatment [12] which likewise may conceivably generalize to the case of complex gauge group.

Both in the case of compact gauge group, studied in [11], and in the case of complex gauge group considered in this paper, the only properties of quantization that are required are the most basic properties, well familiar to quantum field 
theorists, of the representations of the canonical commutation relations (for bosons). A convenient framework for organizing the discussion is provided by elementary concepts and terminology of the theory of geometric quantization $[13,14]$ (for reviews see $[15,16])$. Perhaps future developments will require deeper aspects of this theory. In this paper, though, a substantive use of the notions of geometric quantization is made only briefly in Sect. 6.1.

This paper is written in a considerably more informal style than [11]. However, the considerations are precise and essentially rigorous, even by mathematical standards. (The heuristic considerations in the early part of the paper can be considered as a motivation and explanation for the latter sections, which are precise.)

\section{The Lagrangian}

In the problem to be treated, the connection $\mathscr{A}$ is a one form with values in the Lie algebra $\mathbf{g}_{\mathbb{C}}$ of a complex Lie group $\mathbf{g}_{\mathbb{C}}$. $G$ will denote a real form of $G_{\mathbb{C}}$, which unless otherwise noted with always be a maximal compact subgroup, and $\mathbf{g}$ will denote the Lie algebra of $G$. The action is

$$
\begin{aligned}
I= & \frac{t}{8 \pi} \int_{M} \operatorname{Tr}\left(\mathscr{A} \wedge d \mathscr{A}+\frac{2}{3} \mathscr{A} \wedge \mathscr{A} \wedge \mathscr{A}\right) \\
& +\frac{\bar{t}}{8 \pi} \int_{M} \operatorname{Tr}\left(\bar{A} \wedge d \bar{A}+\frac{2}{3} \bar{A} \wedge \overline{\mathscr{A}} \wedge \overline{\mathscr{A}}\right)
\end{aligned}
$$

with $t$ and $\bar{t}$ being complex parameters. Here $\overline{\mathscr{A}}$ is the complex conjugate of $\mathscr{A}$, and for the Lagrangian to be real (which we will not necessarily require; see below) $\bar{t}$ is the complex conjugate of $t$. Very naively, if one regards $\mathscr{A}$ and $\overline{\mathscr{A}}$ as independent variables, ignoring the fact that they are complex conjugates of each other, than (2.1) looks like a product of two ordinary Chern-Simons theories, with couplings $k^{*}{ }_{1}=t / 2$ and $k^{*}{ }_{2}=\bar{t} / 2$. In this paper, we will determine exactly to what extent and in what respect such a naive statement is correct.

It is convenient to write $t=k+i s, \bar{t}=k-i s$ (with $k$ and $s$ real if $\bar{t}$ is the complex conjugate of $t$ ), and $\mathscr{A}=A+i B$, with $A$ and $B$ being $\mathbf{g}$ valued one forms. The Lagrangian then takes the form

$$
\begin{aligned}
I= & \frac{k}{4 \pi} \int_{M} \operatorname{Tr}\left(A \wedge d A-B \wedge d B+\frac{2}{3} A \wedge A \wedge A-2 A \wedge B \wedge B\right) \\
& -\frac{s}{2 \pi} \int_{M} \operatorname{Tr}\left(A \wedge d B+2 A \wedge A \wedge B-\frac{2}{3} B \wedge B \wedge B\right) .
\end{aligned}
$$

The parameter $k$ is subject to the same quantization law as in Chern-Simons theory of the compact group $G$; if " $T r$ " is normalized correctly (in the case $G=$ $S U(N)$, "Tr" should denote the trace in the $N$ dimensional representation), then $k$ must be an integer. This requirement comes, of course, from requiring singlevaluedness of the integrand in the Feynman path integral

$$
Z=\int \mathscr{D} \mathscr{A} e^{i I} \text {. }
$$


In particular, the topological condition means that $k$ must be real whether or not a reality condition is imposed on the Lagrangian. On the other hand, $s$ is not subject to a topological condition. The reason that $k$ is quantized and not $s$ is that the group $G_{\mathbb{C}}$ is contractible onto its maximal compact subgroup, and therefore the only topological quantization laws that arise are those that come from that subgroup.

Reality Conditions. Now, let us discuss what reality condition must be placed on $s$ in order to obtain a unitary quantum field theory. In Minkowski space, the physical condition is simply that the Lagrangian should be real. This corresponds to $t=\bar{t}$ and thus to real $s$, which should therefore lead to a unitary theory.

We also wish to consider the thornier issue of unitarity in Euclidean space. The physical requirement is that under a reversal of orientation of space-time, the argument of the Euclidean path integral should be complex conjugated. Because of the explicit " $i$ " in the exponent in (2.3), this means that the action $I$ should be odd under reversal of orientation combined with complex conjugation. This is the case if $s$ is real and $A$ and $B$ are invariant under reversal of orientation, since integration of differential forms is odd under reversal of orientation. (In other words, the use of wedge products in (2.2) could be eliminated in favor of an explicit appearance of the Levi-Civita tensor $\varepsilon^{i j k}$, and this is odd under parity.) So we learn again that (2.2) should be expected to define a unitary quantum field theory if $s$ is real. There is, however, a second possibility for how (2.2) can be odd under complex conjugation combined with reversal of orientation. In fact, if we consider $B$ to be odd under reversal of orientation, then for (2.2) to be odd under reversal of orientation combined with complex conjugation, $s$ must be imaginary! Thus, the theory should have a second unitary branch at imaginary $s$. (The statement that $B$ is odd under reversal of orientation means that we are combining a reversal of orientation of $M$ with an involution of $G_{\mathbb{C}}$ that fixes the real form $G$.)

2.1. Specialization to General Relativity. To illustrate these ideas, let us consider the important case of $2+1$ dimensional general relativity. There are two relevant possibilities:

(i) $S L(2, \mathbb{C})$ is the gauge group that describes $2+1$ dimensional gravity in a space-time of Lorentz signature and with a positive cosmological constant. In writing (2.2), it is convenient to take $G$ to be the real form $S L(2, \mathbb{R})$ of $S L(2, \mathbb{C})$. $(S L(2, \mathbb{R})$ is a double cover of the $2+1$ dimensional Lorentz group $S O(2,1)$. Except in this paragraph, we will always in this paper understand $G$ to be a maximal compact subgroup of $G_{\mathbb{C}}$, even when discussing gravity.) With $G=S L(2, \mathbb{R}), A$ can be identified with the spin connection $\omega$ of general relativity, and $B$ with the vierbein $e$. With this identification, the term in (2.2) proportional to $s$ is the Einstein-Hilbert action with a cosmological constant. In particular, $s$ is essentially the inverse of Newton's constant, and the statement that the theory is unitary for real $s$ is simply the statement that general relativity is unitary when Newton's constant is real. The $k$ coupling is a new discrete parameter [4] that is possible in $2+1$ dimensional quantum gravity when formulated as a gauge theory.

(ii) $S L(2, \mathbb{C})$ is also the gauge group that is relevant to Euclidean quantum gravity with negative cosmological constant. In this case, it is natural to take $G=S U(2)$ 
(which is the double cover of the Euclidean rotation group $S O(3)$ ), and to again identify $A$ and $B$ with the spin connection $\omega$ and vierbein $e$. With this interpretation, the inverse of Newton's constant is proportional to is, and therefore $s$ should be imaginary. (The reason that the $i$ comes in is that in Euclidean quantum gravity, the argument of the Feynman path integral should be $\exp (-$ real constant $\left.\int_{M} \sqrt{g} R+\cdots\right)$ rather than $\exp \left(i \cdot\right.$ real constant $\left.\cdot \int_{M} \sqrt{g} R+\cdots\right)$. Since there is an " $i$ " in (2.3), to avoid an unwanted $i$ multiplying the Einstein-Hilbert action in Euclidean quantum gravity, $s$ must be imaginary.) In the general discussion, we noted that imaginary $s$ corresponds to $B$-or $e$-being odd under reversal of orientation. This is also natural in Euclidean gravity. In classical gravity, one considers only vierbeins of positive determinant, that is, such that $\varepsilon^{i j k} \varepsilon_{a b c} e_{i} e_{j}{ }_{j}^{b} e_{k}{ }^{c}>0$. For this condition to be invariant under reversal of orientation (which changes the sign of $\varepsilon^{i j k}$ ), one must consider $e$ to change sign under reversal of orientation.

Thus, in studying (2.2), we will write formulas that depend on a free parameter $s$. The formalism will be developed in a way that is natural for real $s$, and all the formulas will be manifestly unitary if $s$ is real. For gauge group $G_{\mathbb{C}}=S L(2, \mathbb{C})$, the unitary theory at real $s$ will be interpreted as Lorentzian quantum gravity with positive cosmological constant. ${ }^{1}$ After obtaining the final formulas, we will analytically continue to complex values of $s$. The definition of Hilbert space inner products that gives unitarity at real $s$ will not give unitarity when $s$ is not real. However, it might happen that at imaginary $s$, it is possible to find an alternative definition of inner products that will give unitarity. If such an alternative unitary structure exists at imaginary $s$, then (for $G_{\mathbb{C}}=S L(2, \mathbb{C})$ ) we can interpret the resulting theory as Euclidean quantum gravity. In this paper, we will find the alternative unitary structure explicitly in the case of canonical quantization on a Riemann suface of genus one. We will not be able to find the alternative unitary structure explicitly for higher genus. ${ }^{2}$ However, we will find a simple geometric interpretation of the hypothetical alternative unitary structure at imaginary $s$. This interpretation and other aspects of the formalism make it clear that the unitary structure at imaginary $s$ is a $2+1$ dimensional analog of the "complementary series" of unitary representations of $G_{\mathbb{C}}$, while the unitary structure at real $s$ is a $2+1$ dimensional analog of the more straightforward "principal series."

Though general relativity is an important motivation for studying the $G_{\mathbb{C}}$ gauge theory, there actually are many unsolved conceptual problems connected with the relation between general relativity and gauge theory in $2+1$ dimensions. These

\footnotetext{
${ }^{1}$ As noted above, in the rest of this paper, the real form $G$ will always be the maximal compact subgroup of $G_{\mathfrak{C}}$. This is permissible even if we are studying Lorentzian quantum gravity, for it is a simple mathematical fact that the Lagrangian (2.1) can be written as in (2.2) with $G$ an arbitrarily chosen real form of (2.2). However, with $G$ the compact form, $A$ and $B$ do not have the interpretation of the three dimensional spin connection and vierbein in the case of Lorentzian gravity. In the canonical quantization that we will be developing, they probably do have a natural interpretation in terms of the geometry of a two dimensional spatial slice

2 This is analogous to the fact that $[9,11]$ in the case of compact gauge groups, it is easier to understand unitarity explicitly in the case of genus one. There is probably a precise connection between the two problems
} 
problems mostly have to do with the role of non-invertible vierbeins, vierbeins of negative determinant, flat connections that define non-discrete embeddings of the fundamental group, etc. Some of these points have been clarified in [17], and from a completely different point of view in [18], but much remains to be understood. This paper is mainly devoted to studying the $G_{\mathbb{C}}$ theory as a gauge theory. We return to the gravitational interpretation only in Sect. 6.1.

It is very unclear whether one should expect Lorentzian and Euclidean gravity to be related to each other by Wick rotations. If so, the imaginary $s$ theory should go under Wick rotation into a Chern-Simons theory with $S L(2, \mathbb{R}) \times S L(2, \mathbb{R})$ gauge group (which describes Lorentzian quantum gravity with negative cosmological constant), and this would seem to imply that, in contrast to what is presently understood, Chern-Simons theory with $S L(2, \mathbb{R})$ gauge group could not be much more difficult than the $S L(2, \mathbb{C})$ case.

\section{Quantization}

We now turn to the problem of quantization of our basic Lagrangian

$$
\begin{aligned}
I= & \frac{t}{8 \pi} \int_{M} \operatorname{Tr}\left(\mathscr{A} \wedge d \mathscr{A}+\frac{2}{3} \mathscr{A} \wedge \mathscr{A} \wedge \mathscr{A}\right) \\
& +\frac{\bar{t}}{8 \pi} \int_{M} \operatorname{Tr}\left(\overline{\mathscr{A}} \wedge d \overline{\mathscr{A}}+\frac{2}{3} \overline{\mathscr{A}} \wedge \overline{\mathscr{A}} \wedge \overline{\mathscr{A}}\right),
\end{aligned}
$$

on a three manifold of the form $M=\Sigma \times \mathbb{R}^{1}$, with $\Sigma$ being an oriented closed two dimensional surface and $\mathbb{R}^{1}$ being the "time" axis; $\mathscr{A}$ is of course a connection on a $G_{\mathbb{C}}$ bundle $E$ over $M$. We will sometimes expand the connection as $\mathscr{A}=\Sigma \mathscr{A}^{a} \cdot T_{a}$, where $T_{a}$ is a basis of the real Lie algebra $G$ (whose complexification is the Lie algebra of $G_{\mathbb{C}}$ ) which we take to be orthonormal in the sense that $\operatorname{Tr}\left(T_{a} T_{b}\right)=\delta_{a b}$. The $\mathscr{A}^{a}$ are complex valued one forms.

Canonical quantization will associate a Hilbert space $\mathscr{H}$ with the Riemann surface $\Sigma$. General covariance means that $\mathscr{H}$ must (up to a possible projective factor) depend on $\Sigma$ only as a topological surface, with no a priori choice of metric or complex structure. $\mathscr{H}$ will be obtained by quantizing an appropriate symplectic manifold. In fact, the symplectic manifold that must be quantized is simply the moduli space of stationary points of the Lagrangian (3.1), up to gauge transformation. Since the Euler-Lagrange equations derived from (3.1) assert that the connection $\mathscr{A}$ should be flat, the symplectic manifold that must be quantized is the moduli space $\mathscr{M}_{\mathbb{C}}$ of flat $G_{\mathbb{C}}$ connections on $\Sigma$, up to gauge transformation (equivalently, the moduli space of representations of the fundamental group of $\Sigma$ in $G_{\mathbb{C}}$, up to conjugation). $\mathscr{M}_{\mathbb{C}}$ has a $t$ dependent symplectic structure that is deduced in the usual way from the Lagrangian.

In previous investigations of Chern-Simons gauge theory with compact gauge group, the moduli space $\mathscr{M}$ of flat $G$ connections has been quantized. Our task is essentially to carry out a similar quantization of $\mathscr{M}_{\mathbb{C}}$. If there were a completely universal theory of quantization, we would proceed by directly quantizing the finite dimensional moduli space $\mathscr{M}_{\mathbb{C}}$, with the $t$ dependent symplectic structure deduced from (3.1). Since such a theory does not exist, we will (just as in similar 
discussions of quantization of $\mathscr{M}$ ) proceed by regarding $\mathscr{M}_{\mathbb{C}}$ as the symplectic quotient of the space $\mathscr{W}_{\mathbb{C}}$ of all $G_{\mathbb{C}}$ connections on $\Sigma$, by the action of the group of gauge transformations. Quantization of $\mathscr{M}_{\mathbb{C}}$ proceeds by formally quantizing $\mathscr{W}_{\mathbb{C}}$ and then picking out the $G_{\mathbb{C}}$ invariant subspace.

The moduli space $\mathscr{M}_{\mathbb{C}}$ has many remarkable properties. For one thing, it has a family of hyper-kahler structures [19], which is likely to be important in deeper understanding of the $G_{\mathbb{C}}$ gauge theory, but will not be used here. It is also true [19] that after picking a complex structure $J$ on $\Sigma$ and deleting a subspace of rather high codimension (which is usually a permissible step in quantum mechanics) $\mathscr{M}_{\mathbb{C}}$ can be identified (in a $J$ dependent fashion) with the cotangent bundle $T^{*} \mathscr{M}$. In quantization of a cotangent bundle $T^{*} X$, the physical Hilbert space consists of $L^{2}$ functions (or sections of a line bundle) on $X$. One might therefore be inclined to guess from the fact just cited that the physical Hilbert space of the $G_{\mathbb{C}}$ gauge theory would have a $J$ dependent identification with $\Gamma(\mathscr{M}, \mathscr{S})$ where $\mathscr{S}$ is a suitable line bundlet over $\mathscr{M}$ and $\Gamma(\mathscr{M}, \mathscr{S})$ denotes the Hilbert space of $L^{2}$ sections of $\mathscr{S}$. This is indeed the result that we will arrive at, after a fairly straightforward though relatively long discussion which will also give us a description of the crucial $J$ dependence.

3.1. Preliminary Discussion. To begin with, we consider the theory defined by the Lagrangian (3.1) in the gauge $\mathscr{A}_{0}=\overline{\mathscr{A}}_{0}=0$. Before imposing any constraints, the phase space with which we begin is the space $\mathscr{W}_{\mathbb{C}}$ of all $G_{\mathbb{C}}$ connections on $E$. In the standard way, the Lagrangian determines a natural ( $t$ dependent) symplectic structure $\omega$ on $\mathscr{W}_{\mathbb{C}}$.

In quantizing $\mathscr{W}_{\mathbb{C}}$, we must impose the Gauss law constraints

$$
F=\bar{F}=0,
$$

where $F=d \mathscr{A}+\mathscr{A} \wedge \mathscr{A}$ is the $G_{\mathbb{C}}$ field strength, and $\bar{F}$ is its complex conjugate. As in $[9,11]$, we will impose the constraints after quantization; that is, we will formally construct a "big" Hilbert space obtained by quantization of $\mathscr{W}_{\mathbb{C}}$ and then restrict to the subspace annihilated by the constraint operators. We adopt this approach because there is not a universal theory of quantization that would tell us directly how to quantize the physical space that is obtained by imposing (3.2) classically and then dividing by the gauge group.

Though we will really only be using elementary properties of the representations of the canonical commutation relations, the discussion that follows is conveniently organized from a viewpoint familiar in the literature on geometric quantization $[13,14]$ (for reviews see $[15,16]$ ). From this point of view, the first step is to define a "prequantum line bundle" $\mathscr{L}_{\mathrm{pr}}$ over the phase space $\mathscr{W}_{\mathbb{C}} \cdot \mathscr{L}_{\mathrm{pr}}$ is a unitary line bundle with a connection $\lambda$ whose curvature is $d \lambda=-i \omega$. In the problem at hand, $\mathscr{L}_{\mathrm{pr}}$ can be characterized by saying that the commutators of covariant derivatives in $\mathscr{W}_{\mathbb{C}}$, acting on sections of $\mathscr{L}_{\mathrm{pr}}$, are

$$
\begin{aligned}
& {\left[\frac{\delta}{\delta \mathscr{A}_{i}^{a}(z)}, \frac{\delta}{\delta \mathscr{A}_{j}^{b}(w)}\right]=-\frac{t}{8 \pi} \delta^{a b} \varepsilon_{i j} \delta(z, w),} \\
& {\left[\frac{\delta}{\delta \overline{\mathscr{A}}_{i}^{a}(z)}, \frac{\delta}{\delta \overline{\mathscr{A}}_{j}^{b}(w)}\right]=-\frac{\bar{t}}{8 \pi} \delta^{a b} \varepsilon_{i j} \delta(z, w),}
\end{aligned}
$$


with other components vanishing. Here $\varepsilon_{i j}$ is the Levi-Civita tensor density on the oriented surface $\Sigma$.

One next defines a prequantum Hilbert space $\mathscr{H}_{\mathrm{pr}}$ which consists of all square integrable sections of the prequantum line bundle $\mathscr{L}_{\mathrm{pr}}$. Thus, we write $\mathscr{H}_{\mathrm{pr}}=$ $\Gamma\left(\mathscr{W}, \mathscr{L}_{\mathrm{pr}}\right)$, (where $\Gamma(X, \mathscr{S})$ denotes the space of square integrable sections of a line bundle $\mathscr{S}$ over a space $X$ ). Acting on the prequantum Hilbert space, the constraint operators - which are none other than the generators of the gauge group - are

$$
\begin{aligned}
& \mathscr{F}_{a}(z)=D_{i} \frac{\delta}{\delta \mathscr{A}_{i}^{a}}-\frac{t}{8 \pi} F_{z \bar{z} a} \\
& \overline{\mathscr{F}}_{a}(z)=D_{i} \frac{\delta}{\delta \overline{\mathscr{A}}_{i}^{a}}-\frac{\bar{t}}{8 \pi} \bar{F}_{z \bar{z} a} .
\end{aligned}
$$

The gauge invariant subspace of $\mathscr{H}_{\mathrm{pr}}$ is the subspace annihilated by these operators.

3.2. Choice of a Polarization. The prequantum Hilbert space is much bigger than the desired quantum Hilbert space because it consists of wave functions that depend on the coordinates and momenta. Thus, in a mechanics problem with a two dimensional $(p, q)$ phase space, the prequantum Hilbert space would consist of functions $\Psi(p, q)$. Quantization is carried out by choosing a "polarization" and requiring the wave-functions to be independent of half of the variables. In this paper, the phase spaces that we quantize are affine spaces and the polarizations are translation invariant. For instance, in the case of a real polarization, one picks one of the coordinates, say $p$, and defines the quantum Hilbert space $\mathscr{H}$ to be the subspace of the prequantum Hilbert space consisting of wave functions $\Psi(p, q)$ such that

$$
0=\frac{D}{D p} \Psi
$$

In the case of a complex polarization, one picks linear combinations $z=q+i p, \bar{z}=q-i p$, and declares the quantum Hilbert space $\mathscr{H}$ to consist of wave functions $\Psi(p, q)$ such that

$$
0=\left(\frac{D}{D q}+i \frac{D}{D p}\right) \Psi=\frac{D}{D \bar{z}} \Psi .
$$

Clearly, in the first case, the quantum Hilbert space consists of functions $\Psi(q)$, and in the second case it consists of holomorphic functions $\Psi(q+i p)$. (Actually, these "functions" are really sections of the prequantum line bundle $\mathscr{L}_{\mathrm{pr}}$. In the case of a real polarization one can forget about $\mathscr{L}_{\mathrm{pr}}$ in the end, since as a line bundle with connection it is trivial when restricted to the $q$ axis. In the case of a complex polarization one cannot so naturally forget about $\mathscr{L}_{\mathrm{pr}}$.) The physical Hilbert spaces defined by the polarizations used in (3.5) and (3.6) are equivalent since they each give irreducible, unitary representations of the canonical commutation relations.

The advantage of first defining the "big" prequantum Hilbert space and then regarding the quantum Hilbert space as a subspace determined by equations such as (3.5) or (3.6) is that this gives a natural framework for thinking about the behavior under a change of polarization. That will be crucial in the problem at 
hand. The reason that it is crucial is that in the quantization of (3.1), there is no natural choice of polarization.

Gauge Invariance of the Polarization. The most important desideratum in the choice of polarization is that after defining the quantum Hilbert space by equations generalizing (3.5) or (3.6), it should be possible to solve the Gauss law constraints. For our goal is not just to formulate the quantum theory associated with the classical Lagrangian (3.1), but to solve it.

If the choice of polarization is gauge invariant, then the auxiliary equations that define the physical Hilbert space commute with the prequantum gauge generators (3.4). In this case, the constraint operators that must annihilate gauge invariant physical states coincide with the prequantum gauge generators. Those operators are first order differential operators which simply say that up to a known phase, the wave functions are independent of some of the variables.

If we use a polarization that is not gauge invariant, then the prequantum gauge generators do not act on the quantum Hilbert space (since they are not compatible with the auxiliary conditions (3.5) or (3.6) that define the quantum Hilbert space as a subspace of the prequantum Hilbert space, on which the prequantum gauge generators do act). In such a case, the constraint operators, which can be found by quantizing the classical expression $\mathscr{F}=d \mathscr{A}+\mathscr{A} \wedge \mathscr{A}$, differ from the prequantum gauge generators by terms which compensate for the change in polarization under a gauge transformation. The latter terms are second order differential operators on $\mathscr{W}_{\mathfrak{C}}$, so in this situation the Gauss law constraints are a system of second order differential equations. Even in a mechanics problem involving finitely many variables, such a system of equations would be very difficult to solve.

Using a polarization that is not gauge invariant leads to a correct but typically intractable formalism. (An example is the Wheeler-de Witt equation of $2+1$ dimensional general relativity, which arises in a certain polarization that is not gauge invariant.) As our goal is to solve the quantum field theory determined by (3.1), not just to talk about it, we will seek a gauge invariant polarization.

3.3. Choice of Complex Structure. In $2+1$ dimensional gravity with zero cosmological constant, it is possible to pick a gauge invariant polarization that also is invariant under diffeomorphisms of $\Sigma$ [4]. This makes it possible to develop the formalism in a manifestly "background-independent" way. In contrast, in Chern-Simons theory of a compact gauge group, there is no such gauge invariant and diffeomorphism invariant polarization. The best that one can do is to use a gauge invariant polarization that depends on the choice of a complex structure $J$ on $\Sigma$ [1]. With such a polarization, one defines a physical Hilbert space $\mathscr{H}_{J}$ that depends on the choice of $J$. One then finds a projectively flat connection on the space of complex structures which gives a projective identification among the $\mathscr{H}_{J}$ as $J$ varies.

Similarly, if we take a semi-simple complex Lie group $G_{\mathbb{C}}$ as gauge group, there seems to be no completely natural and gauge invariant polarization. By analogy with the case of a compact gauge group, we will treat this problem by choosing a polarization that is gauge invariant but depends on the choice of a complex structure on $\Sigma$. As $J$ varies, the quantum Hilbert spaces $\mathscr{H}_{J}$ then define a "quantum 
bundle" $\mathscr{H}_{Q}$ over the space $\mathscr{J}$ of possible complex structures. We must then find a projectively flat connection on this quantum bundle, permitting us to say that the quantization is in fact independent of the choice of $J .^{3}$ This will be done using the methods of [11].

Defining the Polarization. We now pick a complex structure $J$ on $\Sigma$. Once $J$ is pickęd, the connection form can locally be written $\mathscr{A}^{a}=\mathscr{A}_{z}{ }^{a} d z+\mathscr{A}_{\bar{z}}{ }^{a} d \bar{z}$, with $z$ being a local complex parameter. Likewise, $\overline{\mathscr{A}}^{a}=\overline{\mathscr{A}}_{z}^{a} d z+\overline{\mathscr{A}}_{\bar{z}}^{a} d \bar{z}$. Since the $\mathscr{A}^{a}$ are complex valued one forms, the $\mathscr{A}_{z}^{a}$ and $\mathscr{A}_{\bar{z}}{ }^{a}$ (with $a=1 \cdots \operatorname{dim} G, G$ being the compact Lie group whose complexification is the gauge group $G_{\mathbb{C}}$ of our theory) are independent complex variables. On the other hand, as $\overline{\mathscr{A}}^{a}$ is the complex conjugate of $\mathscr{A}^{a}, \overline{\mathscr{A}}_{z}^{a}$ is the complex conjugate of $\mathscr{A}_{\bar{z}}{ }^{a}$, and $\overline{\mathscr{A}}_{\bar{z}}{ }^{a}$ is the complex conjugate of $\mathscr{A}_{z}{ }^{a}$.

If $J$ is oriented correctly, which we now require, the commutation relations (3.3) that characterize the prequantum line bundle become

$$
\begin{aligned}
& {\left[\frac{\delta}{\delta \mathscr{A}_{z}^{a}(z)}, \frac{\delta}{\delta \mathscr{A}_{\bar{z}}^{b}(w)}\right]=-\frac{t}{8 \pi} \delta^{a b} \delta(z, w),} \\
& {\left[\frac{\delta}{\delta \mathscr{\mathscr { A }}_{z}^{a}(z)}, \frac{\delta}{\delta \overline{\mathscr{A}}_{\bar{z}}^{b}(w)}\right]=-\frac{\bar{t}}{8 \pi} \delta^{a b} \delta(z, w) .}
\end{aligned}
$$

A vector in the prequantum Hilbert space $\mathscr{H}_{0}$ is, formally, an $L^{2}$ section $\Psi\left(\mathscr{A}_{z}^{a}, \mathscr{A}_{\bar{z}}^{b}\right)$ of the prequantum line bundle $\mathscr{L}_{\mathrm{pr}}$; it is, of course, not required to be holomorphic or antiholomorphic as a function of these variables. To quantize, we now introduce a real polarization of the space $\mathscr{W}_{\mathbb{C}}$ of complex connections by saying that the real and imaginary parts of $\mathscr{A}_{\bar{z}}{ }^{a}$ will be regarded as coordinates, and the real and imaginary parts of $\mathscr{A}_{z}{ }^{a}$ will be regarded as momenta. This choice defines a valid polarization because the real and imaginary part of $\mathscr{A}_{\bar{z}}{ }^{a}$ have vanishing Poisson brackets with one another, and are canonically conjugate to the real and imaginary parts of $\mathscr{A}_{z}^{a}$. With this polarization, the $J$ dependent quantum Hilbert space $\mathscr{H}^{\prime}{ }_{J}$ is the subspace of $\mathscr{H}_{0}$ consisting of wave functions that obey

$$
0=\frac{\delta}{\delta \mathscr{A}_{z}^{a}} \Psi=\frac{\delta}{\delta \overline{\mathscr{A}}_{\bar{z}}{ }^{a}} \Psi .
$$

(As $\overline{\mathscr{A}}_{\bar{z}}{ }^{a}$ is the complex conjugate of $\mathscr{A}_{z}^{a},(3.8)$ is the statement that $\Psi$ is annihilated by the covariant derivatives with respect to the real or imaginary part of $\mathscr{A}_{z}^{a}$.)

In this paper, the full quantum Hilbert space, without imposing a constraint of gauge invariance, will be denoted as $\mathscr{H}^{\prime}$ or as $\mathscr{H}^{\prime}{ }_{J}$ to emphasize its $J$ dependence; its gauge invariant subspace will be called simply $\mathscr{H}$ or $\mathscr{H}_{\mathrm{J}}$, the physical Hilbert space. Similarly, the quantum connection on $\mathscr{H}^{\prime}$ will be called $\delta^{\prime} Q$, or just $\delta^{\prime}$, while the quantum connection on the gauge invariant subspace $\mathscr{H}$ will be called $\delta^{\mathcal{Q}}$.

\footnotetext{
${ }^{3}$ It is interesting to note that $2+1$ dimensional general relativity with positive cosmological constant, like string theory, is a generally covariant theory which is very difficult to formulate in a background independent way. In the former case background dependence of an unusual kind enters in the choice of a polarization which is needed for quantization. In the latter case we still do not understand the origin of the background dependence which is so prominent in presently known formulations
} 
The quantum Hilbert space $\mathscr{H}^{\prime}{ }_{J}$ depends on $J$ because Eqs. (3.8) depend on $J$. It is convenient to make the dependence on $J$ explicit and to think of the quantum wave functional $\Psi\left(\mathscr{A}_{i} ; J\right)$ as a functional of $\mathscr{A}$ and $J$ which for any given $J$ obeys (3.8). We would like to write down an equation describing how $\Psi\left(\mathscr{A}_{i} ; J\right)$ must vary as $J$ varies so that the quantum state represented by $\Psi$ is independent of $J$. The nature of this can be understood along the following lines. The representation of the canonical commutation relations that we are using (with $\Psi$ depending only on the coordinates $\mathscr{A}_{\bar{z}}$ and not on the momenta $\mathscr{A}_{z}$ ) depends on $J$. As $J$ varies, one must make a Bogoliubov transformation on the wave function to compensate for the change in representation of the commutation relations. The generator of a Bogoliubov transformation is quadratic in the coordinates and momenta, so it will be represented by a second order differential operator in the equation that we will write.

Let $\mathscr{J}$ denote the space of complex structures on $\Sigma . \mathscr{J}$ is a complex rnanifold, so the exterior derivative $\delta$ on $\mathscr{J}$ has a decomposition $\delta=\delta^{(1,0)}+\delta^{(0,1)}$ with the two pieces being of type $(1,0)$ and $(0,1)$, respectively. Under a change in complex structure of $\Sigma$, one has

$$
\begin{array}{rlrl}
\delta^{(1,0)}\left(\frac{\delta}{\delta \mathscr{A}_{z}^{a}}\right) & =\frac{i}{2} \delta J^{z}{ }_{\bar{z}} \frac{\delta}{\delta \mathscr{A}_{\bar{z}}^{a}}, & \delta^{(0,1)}\left(\frac{\delta}{\delta \mathscr{A}_{z}^{a}}\right)=0, \\
\delta^{(0,1)}\left(\frac{\delta}{\delta \overline{\mathscr{A}}_{\bar{z}}^{a}}\right)=-\frac{i}{2} \delta J^{\bar{z}}{ }_{z} \frac{\delta}{\delta \overline{\mathscr{A}}_{z}^{a}}, & \delta^{(0,1)}\left(\frac{\delta}{\delta \overline{\mathscr{A}}_{\bar{z}}^{a}}\right)=0,
\end{array}
$$

where $\delta J^{z}{ }_{\bar{z}}$ and $\delta J^{\bar{z}}{ }_{z}$ can be regarded as forms of type $(1,0)$ and $(0,1)$ on $\mathscr{J}$, respectively. The desired connection that expresses how $\Psi(\mathscr{A} ; J)$ must vary as $J$ changes in order to describe a fixed quantum state is (by analogy with Eq. (2.37 of [11]) formally

$$
\begin{aligned}
\delta^{(1,0)} & =\delta^{(1,0)}-\frac{2 i \pi}{t} \int_{\Sigma} \delta J_{\bar{z}}^{z} \frac{\delta}{\delta \mathscr{A}_{\bar{z}}^{a}} \frac{\delta}{\delta \mathscr{A}_{\bar{z}}^{a}}, \\
\delta^{(0,1)} & =\delta^{(0,1)}-\frac{2 i \pi}{\bar{t}} \int_{\Sigma} \delta J^{\bar{z}}{ }_{z} \frac{\delta}{\delta \overline{\mathscr{A}}_{z}{ }^{a}} \frac{\delta}{\delta \overline{\mathscr{A}}_{z}^{a}} .
\end{aligned}
$$

The condition that the quantum state represented by a wave function $\Psi(\mathscr{A} ; J)$ is independent of $J$ is simply

$$
\delta^{\prime} \Psi=0 .
$$

This equation makes sense because, using (3.7) and (3.9), one can verify that

$$
\left[\frac{\delta}{\delta \mathscr{A}_{z}^{a}}, \delta^{\prime}\right]=\left[\frac{\delta}{\delta \overline{\mathscr{A}}_{\bar{z}}^{a}}, \delta^{\prime}\right]=0 .
$$

This ensures that a physical state $\Psi$, which obeys (3.8) at one value of $J$, still obeys (3.8) (and thus still represents a physical state) after being transported via the connection $\delta^{\prime}$. Given that $\delta^{\prime}$ differs from $\delta$ by a second order differential operator, the form of $\delta^{\prime}$ is uniquely determined by requiring (3.12).

Having quantized the theory, we now wish to impose the Gauss's law constraints and select the gauge invariant subspace of the quantum Hilbert space. 
In our chosen polarization, the generators (3.4) of the gauge group become

$$
\begin{aligned}
& \mathscr{F}_{a}(z)=D_{\bar{z}} \frac{\delta}{\delta \mathscr{A}_{\bar{z}}^{a}}-\frac{t}{8 \pi} F_{z \bar{z} a}, \\
& \overline{\mathscr{F}}_{a}(z)=D_{z} \frac{\delta}{\delta \overline{\mathscr{A}}_{z}{ }^{a}}-\frac{\bar{t}}{8 \pi} F_{z \bar{z} a} .
\end{aligned}
$$

For given $J$, a gauge invariant physical state is a functional $\Psi(\mathscr{A} ; J)$ that obeys (3.8) and (3.13). Such a functional represents a $J$-independent quantum state if it also obeys (3.11). These equations are very similar to the equations that enter in quantizing Chern-Simons gauge theory with a compact gauge group, and they can be studied using the same methods.

3.4. Reduction to the Space of Unitary Connections. Before proceeding further, it is convenient to solve Eq. (3.8), using them to reduce the problem from the space $\mathscr{W}_{\mathbb{C}}$ of complex connections with structure group $G_{\mathbb{C}}$ to the space $\mathscr{W}$ of unitary connections with structure group the maximal compact subgroup $G$.

To do so, we write $\mathscr{A}=A+i B$, with $A$ and $B$ being $\mathbf{g}$ valued one forms (thus $A$ and $B$ are the hermitian and anti-hermitian parts of the $\mathbf{g}_{\mathbb{C}}$-valued one form $\mathscr{A})$. We then have

$$
\begin{gathered}
\frac{\delta}{\delta \mathscr{A}_{z}}=\frac{1}{2}\left(\frac{\delta}{\delta A_{z}}-i \frac{\delta}{\delta B_{z}}\right), \\
\frac{\delta}{\delta \overline{\mathscr{A}}_{\bar{z}}}=\frac{1}{2}\left(\frac{\delta}{\delta A_{\bar{z}}}+i \frac{\delta}{\delta B_{\bar{z}}}\right), \\
\frac{\delta}{\delta \mathscr{A}_{\bar{z}}}=\frac{1}{2}\left(\frac{\delta}{\delta A_{\bar{z}}}-i \frac{\delta}{\delta B_{\bar{z}}}\right), \\
\frac{\delta}{\delta \overline{\mathscr{A}}_{z}}=\frac{1}{2}\left(\frac{\delta}{\delta A_{z}}+i \frac{\delta}{\delta B_{z}}\right) .
\end{gathered}
$$

So the physical state condition (3.8) becomes

$$
\frac{\delta}{\delta B_{z}} \Psi=-i \frac{\delta}{\delta A_{z}} \Psi, \frac{\delta}{\delta B_{\bar{z}}} \Psi=+i \frac{\delta}{\delta A_{\bar{z}}} \Psi
$$

Clearly (3.15) is just sufficient to determine the $B$ dependence of $\Psi$ and thus to determine $\Psi(A, B ; J)$ in terms of $\Psi(A, 0 ; J)$. Moreover, from (3.14) it follows that if $\Psi$ obeys (3.8), then

$$
\frac{\delta}{\delta \mathscr{A}_{\bar{z}}} \Psi=\frac{\delta}{\delta A_{\bar{z}}} \Psi, \quad \frac{\delta}{\delta \overline{\mathscr{A}}_{z}} \Psi=\frac{\delta}{\delta A_{z}} \Psi
$$

This last equation permits us to rewrite the formulas derived above with $B$ eliminated and thereby to formulate the entire discussion in terms of $\Psi(A, 0 ; J)$, which we will henceforth call simply $\Psi(A ; J)$. In fact, the connection becomes 


$$
\begin{aligned}
& \delta^{(1,0)}=\delta^{(1,0)}-\frac{2 i \pi}{t} \int_{\Sigma} \delta J^{z}{ }_{\bar{z}} \frac{\delta}{\delta{A_{\bar{z}}}^{a}} \frac{\delta}{\delta{A_{\bar{z}}}^{a}} \\
& \delta^{(0,1)}=\delta^{(0,1)}-\frac{2 i \pi}{\bar{t}} \int_{\Sigma} \delta J^{\bar{z}}{ }_{z} \frac{\delta}{\delta A_{z}^{a}} \frac{\delta}{\delta{A_{z}}^{a}}
\end{aligned}
$$

Likewise, the constraint generators (3.13) become

$$
\begin{aligned}
& \mathscr{F}_{a}(z)=D_{\bar{z}} \frac{\delta}{\delta A_{\bar{z}}{ }^{a}}-\frac{t}{8 \pi} F_{z \bar{z} a}, \\
& \overline{\mathscr{F}}_{a}(z)=D_{z} \frac{\delta}{\delta A_{z}{ }^{a}}-\frac{\bar{t}}{8 \pi} F_{z \bar{z} a} .
\end{aligned}
$$

In terms of $A$, the commutation relations (3.7) become

$$
\left[\frac{\delta}{\delta A_{z}{ }^{a}(z)}, \frac{\delta}{\delta A_{\bar{z}}{ }^{b}(w)}\right]=-\frac{k}{4 \pi} \delta^{a b} \delta(z, w) .
$$

Here $k=(t+\bar{t}) / 2$ must be an integer for topological reasons.

By now, all of the essential equations have been expressed as equations on the space $\mathscr{W}$ of unitary connections. This is the same space that arises in studying Chern-Simons gauge theory with compact gauge group. Moreover, the commutation relations (3.19) also arise in that context; their geometrical meaning is that the quantum wave functional $\Psi$ is a section of a line bundle $\mathscr{L}^{\otimes k}$, with $\mathscr{L}$ being the fundamental prequantum line bundle over $\mathscr{W}$. It was discussed extensively in $[11] .{ }^{4}$ From the point of view that we are developing here, the essential difference between the compact and complex gauge groups is that in the case that the gauge group is the compact group $G$, the quantum Hilbert space consists of holomorphic sections of $\mathscr{L}^{\otimes k}$. But with gauge group $G_{\mathbb{C}}$, the quantum Hilbert space consists of arbitrary $L^{2}$ sections of $\mathscr{L}^{\otimes k}$.

Because of this difference, the $(0,1)$ part of the connection, which is trivial in the case of gauge group $G$, is non-trivial in the case that the gauge group is $G_{\mathbb{C}}$.

Likewise, the fact that the wave functions are $L^{2}$ rather than holomorphic sections of $\mathscr{L}^{\otimes k}$ is the reason that the constraints (3.18) do not quite coincide with the Gauss's law constraints that arise in quantizing Chern-Simons gauge theory with a compact gauge group. The constraints (3.18) nevertheless play a simple role quite similar to the role that they play in the case of compact gauge group. Let $\hat{G}_{\mathbb{C}}$ be the group of smooth maps from $\Sigma$ to $G_{\mathbb{C}}$, with pointwise multiplication. Then the space $\mathscr{W}$ of unitary connections admits (once a complex structure $J$ is picked) a natural $\hat{G}_{\mathbb{C}}$ action. Indeed, the action of $\hat{G}_{\mathbb{C}}$ by conjugation on the operator $\bar{\partial}_{A}$ (that is, $\bar{\partial}_{A} \rightarrow g \bar{\partial}_{A} g^{-1}$ ) corresponds to a $\hat{G}_{\mathbb{C}}$ action on the space $\mathscr{W}$ of unitary connections. The properly defined quotient of $\mathscr{W}$ by the $\hat{G}_{\mathbb{C}}$ action is simply the moduli space $\mathscr{M}_{J}$ of stable holomorphic $G_{\mathbb{C}}$ bundles over $\Sigma$. The action of $\hat{G}_{\mathbb{C}}$ on $\mathscr{W}$ gives a projection map (defined on a dense open set, which is good

\footnotetext{
${ }^{4}$ Thus, the prequantum line bundle $\mathscr{L}_{\mathrm{pr}}$, when it is restricted to $\mathscr{W}$, becomes isomorphic to $\mathscr{L}^{\otimes k}$. When pushed down to the moduli space $\mathscr{M}_{\mathbb{C}}$, the topology of $\mathscr{L}_{\mathrm{pr}}$ is determined by $k$, but its connection and curvature of course depend on the complex parameter $t$
} 
enough in the $L^{2}$ theory considered here) $\pi: \mathscr{W} \rightarrow \mathscr{M}_{\mathrm{J}}$. The line bundle $\mathscr{L}$ over $\mathscr{W}$ admits a $\hat{G}_{\mathbb{C}}$ action, and pushes down to a line bundle $\mathscr{L}_{J}$ over the moduli space $\mathscr{M}_{J}$.

The constraints (3.18) mean that a physical state in the $G_{\mathbb{C}}$ theory must be invariant under the $\hat{G}_{\mathbb{C}}$ action on $\mathscr{W}$, or more exactly, under a suitable lift of this action to an action on the line bundle $\mathscr{L}^{\otimes k}$. Since a $G_{\mathbb{C}}$ invariant element of $\Gamma\left(\mathscr{W}, \mathscr{L}^{\otimes k}\right)$ is the same as the pullback (via the projection $\pi$ ) of an element of $\Gamma\left(\mathscr{M}_{J}, \mathscr{L}_{J}^{\otimes k}\right)$, we see that the physical Hilbert space of the $G_{\mathbb{C}}$ theory can be identified with $\Gamma\left(\mathscr{M}_{J}, \mathscr{L}_{J}{ }^{\otimes k}\right)$.

According to the Narasimhan-Seshadri theorem, as discussed for instance in [11], the spaces $\mathscr{M}_{J}$ are as symplectic manifolds canonically independent of $J$, and can be identified with the moduli space $\mathscr{M}$ of flat $G$ connections on the surface $\Sigma$. Likewise, the line bundle $\mathscr{L}_{J}$, as a unitary line bundle with connection, is naturally independent of $J$; it can be regarded as a line bundle, which we will call $\mathscr{L}$, defined once and for all on $\mathscr{M}$. We have learned that the physical Hilbert space of the $G_{\mathbb{C}}$ theory has a $J$ dependent identification with the fixed Hilbert space $\mathscr{H}=\Gamma\left(\mathscr{M}, \mathscr{L}^{\otimes k}\right)$.

Therefore, the quantum Hilbert space bundle $\mathscr{H}_{Q}$ over the space $\mathscr{J}$ of complex structures is naturally trivial; and it could be given a natural, trivial flat connection. This possibility is realized in the $G_{\mathbb{C}}$ gauge theory in the limit of $t, \bar{t} \rightarrow \infty$ (with fixed $k$ ), since we see in (3.17) that the connection $\delta^{\prime}$ reduces in that limit to the trivial connection $\delta$. But in general, the connection that must be understood to understand the $G_{\mathbb{C}}$ theory is not the trivial connection but a one parameter generalization of it, depending on a parameter $t$. In the next section, we will use the constraints (3.18) to reduce the connection to a concrete and manageable form on the finite dimensional manifold $\mathscr{M}$.

\section{Concrete Form of the Connection}

At this point, the quantum states in the $G_{\mathbb{C}}$ gauge theory are functionals $\Psi \in \Gamma\left(\mathscr{W} \times \mathscr{J}, \mathscr{L}{ }^{\otimes k}\right)$ (we recall that $\mathscr{W}$ is the space of unitary, $G$-valued connections and $\mathscr{J}$ is the space of complex structures on $\Sigma$ ) which are annihilated by the connection

$$
\begin{aligned}
& \delta^{(1,0)}=\delta^{(1,0)}-\frac{2 i \pi}{t} \int_{\Sigma} \delta J^{z}{ }_{\bar{z}} \frac{\delta}{\delta{A_{\bar{z}}}^{a}} \frac{\delta}{\delta{A_{\bar{z}}}^{a}}, \\
& \delta^{(0,1)}=\delta^{(0,1)}-\frac{2 i \pi}{\bar{t}} \int_{\Sigma} \delta J^{\bar{z}}{ }_{z} \frac{\delta}{\delta A_{z}^{a}} \frac{\delta}{\delta{A_{z}}^{a}},
\end{aligned}
$$

and also obey

$$
\begin{aligned}
& \left(D_{\bar{z}} \frac{\delta}{\delta A_{\bar{z}}{ }^{a}}-\frac{t}{8 \pi} F_{z \bar{z} a}\right) \Psi=0 \\
& \left(D_{z} \frac{\delta}{\delta A_{z}{ }^{a}}-\frac{\bar{t}}{8 \pi} F_{z \bar{z} a}\right) \Psi=0 .
\end{aligned}
$$

Of course, it is unsatisfactory to have an allegedly soluble quantum field theory formulated in terms of functions on the infinite dimensional space $\mathscr{W} \times \mathscr{J}$. Our 
next task is to describe precisely how the Gauss law constraints (4.2) reduce the problem from the infinite dimensional space $\mathscr{W}$ to the finite dimensional space $\mathscr{M}=\mathscr{W} / \hat{G}_{\mathbb{C}}$.

We will follow conventions of [11] concerning the differential geometry of $\mathscr{M}$. In particular, the choice of a complex structure $J$ on $\Sigma$ determines a complex structure on $\mathscr{M}$ that we will also call $J$; it can be represented explicitly as a tensor $J^{i}{ }_{j}$. We project out the $(1,0)$ and $(0,1)$ components of vector fields on $\mathscr{M}$ by writing, for instance, $v^{i}=\frac{1}{2}\left(\delta^{i}{ }_{j}-i J^{i}{ }_{j}\right) v^{j}, v^{\bar{i}}=\frac{1}{2}\left(\delta^{i}{ }_{j}+i J^{i}{ }_{j}\right) v^{j}$, and similarly for tensor fields of other type. The symplectic structure of $\mathscr{M}$ is $\omega_{i j}=k \cdot \omega_{0 i j}$, with $k$ an integer and $\omega_{0}$ the fundamental quantizable symplectic structure; the inverse of $\omega_{0}$ is denoted as $\omega_{0}{ }^{j k}$. We also make the frequent abbreviation $\delta J^{i j}=\delta J^{i}{ }_{k} \omega_{0}{ }^{k j}$.

Another basic object that enters is the determinant of the Laplacian on $\Sigma$, in the adjoint representation of $G$. Thus, if we are given a metric on $\Sigma$, and a connection $A$ on a $G$ bundle $E$, then we have a Laplacian $\triangle=-g^{z \bar{z}}\left(D_{z} D_{\bar{z}}+D_{\bar{z}} D_{z}\right)$. Its determinant, defined with zeta function regularization [20], will be denoted as $H$. $H$ is not quite invariant under a conformal rescaling of the metric of $\Sigma$; under such a rescaling, $H$ changes by a conformal anomaly term that is independent of $A$.

The essential point to be noted is that the first equation in (4.1) and the first equation in (4.2) are precisely the key equations that appear in studying Chern-Simons gauge theory with a compact gauge group, except that where $k$ appears in the problem with gauge group $G$, it is replaced by $t / 2$ in the $G_{\mathbb{C}}$ case. Thus, the first equation in (4.1) corresponds to Eq. (2.37) of [11], and the first equation in (4.2) corresponds to Eq. (2.21) of [11]. The main steps in [11] were the use of the Gauss law constraint, the first part of our Eq. (4.2), to reduce the "upstairs" connection, the first part of our (4.1), to a concrete form given in Eq. (2.42) of [11], and then clarified in Eq. (2.49) and (2.50) of that paper. Basically, in this derivation one uses the first equation in (4.2) to "solve" for $\delta / \delta A_{\bar{z}}$, and then one substitutes the result in (4.1) to simplify the latter. T'1e "downstrairs" connection resulting from this analysis was expressed purely in terms of the differential geometry of $\mathscr{M}$ and the functional determinant $H$ in Eq. (3.36) of [11]. We may simply borrow the latter formula, with $k$ replaced by $t / 2$.

Before writing down the result, let us note the following points:

(i) In the derivation in [11], one meets Green's functions on the diagonal which must be regularized. The proper incorporation of the resulting "anomalies" was described in [11] (cf. the analogous discussion in [9]). The role played by the anomalies in the theory with gauge group $G_{\mathbb{C}}$ is somewhat different, since the conditions that must be satisfied are different. We will therefore first write down the formal expressions and then consider the effects of anomalies.

(ii) Before using the Gauss's law constraints to simplify (4.1), the quantum wave function $\Psi$ is defined on $\mathscr{W} \times \mathscr{J}$, with $\mathscr{J}$ the space of all (properly oriented) complex structures on $\Sigma$. After using the Gauss's law constraints, $\mathscr{W}$ can be replaced by the moduli space $\mathscr{M}$ of flat connections, and since the resulting formulas are invariant under diffeomorphisms that are continuously connected to the identity, the formulas descend from the space $\mathscr{J}$ of all complex structures to Teichmuller space $\mathscr{T}$, which classifies complex structures up to isotopy. Thus, we will henceforth be working on a finite dimensional space $\mathscr{M} \times \mathscr{T}$.

Borrowing the essential results from [11] and replacing $k$ by $t / 2$, we learn that 
the $(1,0)$ part of the connection is formally

$$
\left.\delta^{Q_{0}(1,0)}=\delta^{(1,0)}+\frac{1}{2 t}\left(\nabla_{\underline{\underline{i}}} \delta J^{\underline{\underline{i}} \underline{\underline{j}}} \nabla_{\underline{\underline{j}}}+\delta J^{\underline{i} \underline{j}}\left(\nabla_{\underline{\underline{i}}} \ln H\right) \nabla_{\underline{\underline{j}}}\right)\right)+\frac{1}{2} \delta^{(1,0)} \ln H
$$

(The connection on the quantum Hilbert space was called $\delta^{\mathscr{H}_{Q}}$ in [11]. We will here shorten the name to $\delta^{Q}$, for quantum connection. The subscript " 0 " in $\delta^{Q}{ }_{0}$ in (4.3) reflects the fact that the considerations are still formal, and we will have to make a modification to take account of anomalies.)

In the case of compact gauge group, the $(0,1)$ part of the connection is trivial. However, for complex gauge group, we must now go on and use the second Gauss's law constraint equation in (4.2) to push down the formula for the $(0,1)$ part of the connection, which is the second equation in (4.1). The computation is a mirror image of the analysis of the $(1,0)$ part of the connection, and the result is

$$
\left.\delta^{Q_{0}(0,1)}=\delta^{(0,1)}-\frac{1}{2 \bar{t}}\left(\nabla_{\bar{l}} \delta J^{l j} \nabla_{\bar{j}}+\delta J^{i j}\left(\nabla_{\bar{l}} \ln H\right) \nabla_{\bar{j}}\right)\right)+\frac{1}{2} \delta^{(0,1)} \ln H
$$

The formulas (4.3) and (4.4) are to be understood as follows. The wave functions are sections of $\mathscr{L}^{\otimes k}$ over $\mathscr{M} \times \mathscr{T}$ which are square integrable when restricted to $\mathscr{M} \times t$ for fixed $t \in \mathscr{T}$. Such wave functions may be regarded as sections of a Hilbert space bundle over $\mathscr{T}$; actually, this is the trivial Hilbert space bundle whose fiber is the fixed Hilbert space $\Gamma\left(\mathscr{M}, \mathscr{L}^{\otimes k}\right)$. On that trivial Hilbert space bundle, (4.3) and (4.4) are a one parameter family of connections which reduce for $t \rightarrow \infty$ to the trivial connection.

Incorporation of Anomalies; Vanishing of the $(2,0)$ Curvature. The next urgent task is to properly incorporate the anomalies and get the correct final formulas; the derivation of (4.3) and (4.4) has involved some formal steps borrowed from [11]. In the case of compact gauge group, the anomalies require some slight modifications of the formal expressions. These modifications were found in [11] by requiring that the connection should commute with the equation analogous to (3.8) which defined the polarization. For complex gauge group, we must expect some analogous modifications of the formulas due to anomalies, but these modifications cannot be found in the same way, since we have already solved (3.8). Also we cannot use physical arguments of the type used in [9], since a $1+1$ dimensional analog of the $G_{\mathbb{C}}$ theory is not presently understood. We will simply find the required modification of (4.3) and (4.4) by demanding that the connection $\delta^{Q}$ that we finally adopt should be projectively flat. We will further assume that (as in the case of compact gauge group) the effect of the anomalies will be to change some of the coefficients in (4.3) and (4.4) while leaving the form of the various terms unchanged.

The first step is to require the vanishing of the $(2,0)$ part of the curvature. Again, the analysis is not difficult since the key elements can be borrowed from [11]. In the analysis of the $(2,0)$ part of the curvature for compact gauge groups in [11], certain identities were discovered which were equivalent to the statement that the operator

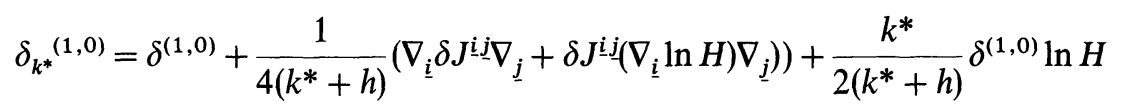


obeys

$$
\left(\delta_{k^{*}}^{(1,0)}\right)^{2}=0
$$

for any complex number $k^{*}$. (Here $h$ is the dual Coxeter number of $G$, so for instance $h=N$ for $G=S U(N)$.) Applying the results of [11] for complex values of $k^{*}$ may require some explanation. In [11], (4.5) was naturally regarded as an operator on sections of the line bundle $\mathscr{L}^{\otimes k^{*}}$ over $\mathscr{M}$, and thus $k^{*}$ was required to be an integer (and was called $k$ ). The standard connection on $\mathscr{L}^{\otimes k^{*}}$ was used in the covariant derivatives $\nabla_{\underline{i}}$, etc., appearing in (4.5). However, since only derivatives of type $(1,0)$ appear in $(4.5)$, and since the curvature of $\mathscr{L}$ with its standard connection is of type $(1,1)$, the line bundle $\mathscr{L}^{\otimes k^{*}}$ plays no role in the analysis of the statement (4.6). We could just as well consider $\delta_{k^{*}}{ }^{(1,0)}$ to be acting on the trivial line bundle (with trivial connection). With this formulation, continuation of $\delta_{k^{*}}^{(1,0)}$ away from integral $k$ makes sense, and it is evident upon examining the relevant discussion in sect. $4(\mathrm{~b})$ of $[11]$ that the vanishing of $(2,0)$ curvature for Chern-Simons gauge theory with compact gauge group is equivalent to the assertion (4.6).

If the discussion in [11] had been carried out with a reversed complex structure on $\Sigma$ and on $\mathscr{M}$, then to verify the vanishing of the $(0,2)$ curvature one would have used the fact that the operator

$$
\left.\delta_{k^{*}}^{(0,1)}=\delta^{(0,1)}+\frac{1}{4\left(k^{*}+h\right)}\left(\nabla_{\bar{l}} \delta J^{\overline{l j}} \nabla_{\bar{j}}+\delta J^{\bar{j} j}\left(\nabla_{\bar{l}} \ln H\right) \nabla_{\bar{j}}\right)\right)+\frac{k^{*}}{2\left(k^{*}+h\right)} \delta^{(0,1)} \ln H
$$

obeys the identity that follows from (4.6) upon reversal of complex structure, namely

$$
\left(\delta_{k^{*}}^{(0,1)}\right)^{2}=0 .
$$

Final Form of the Connection. Comparing (4.3) to (4.5), we see that there is no value of $k^{*}$ for which these coincide, and therefore the $(2,0)$ curvature of the naive connection $\delta^{Q}{ }_{0}$ defined in (4.3) is not zero. We must therefore make some modification of (4.3), to reflect the "anomalies." If vanishing of the $(2,0)$ curvature is the only desideratum, the solution of this problem is not unique, since one could simply replace (4.3) by (4.5), for arbitrary $k^{*}$. However, we also want the non-central part of the $(1,1)$ curvature to vanish, and in the investigation of this point (see the last part of this section) one learns that it is essential that the coefficient of the second order operator $\nabla_{\underline{i}} \delta J^{\underline{i}-\underline{j}} \nabla_{\underline{j}}$ in (4.3) should not be modified. ${ }^{5}$ This forces us to make the choice $k^{*}+h=t / 2$.

So we arrive at the final form of the $(1,0)$ part of the connection:

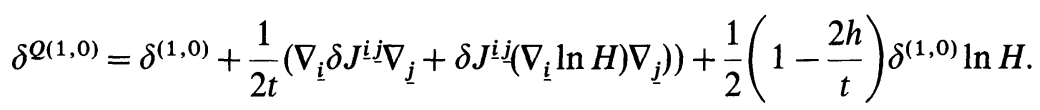

\footnotetext{
${ }^{5}$ The fact that this coefficient retains the value given by the formal manipulation is presumably related to the vanishing of the canonical line bundle of $\mathscr{M}_{\mathbb{C}}$, but we will not attempt to develop that point of view here
} 
Now we require the analogous modification of the $(0,1)$ part of the connection. To ensure vanishing of the $(0,2)$ part of the curvature, we modify the preliminary form of the connection in (4.4) so that it coincides with (4.7) for some value of $k^{*}$, again in such a way that the second order term in the preliminary expression for the connection is unmodified. Evidently, we must pick $k^{*}$ such that $k^{*}+h=-\bar{t} / 2$. Upon doing this, we arrive at the final at the final form of the $(0,1)$ part of the connection:

$$
\left.\delta^{Q(0,1)}=\delta^{(0,1)}-\frac{1}{2 \bar{t}}\left(\nabla_{\bar{l}} \delta J^{\bar{i} j} \nabla_{\bar{j}}+\delta J^{\overline{i j}}\left(\nabla_{\bar{l}} \ln H\right) \nabla_{\bar{j}}\right)\right)+\frac{1}{2}\left(1+\frac{2 h}{\bar{t}}\right) \delta^{(0,1)} \ln H .
$$

The discussion in this paper up to this point may appear somewhat heuristic; in particular, the ad hoc modification of the connection to ensure that the $(2,0)$ and $(0,2)$ parts of the curvature vanish is quite unsatisfying. However, once we arrive at the formulas (4.9) and (4.10) which define the connection, the remainder of the analysis is perfectly precise and rigorous. To emphasize this point for mathematical readers, I will now state the main result of this paper as a theorem. In doing so, we recall that

$$
t=k+i s, \quad \bar{t}=k-i s,
$$

where $k$ is an integer and $s$ is a complex number which is real in the Lorentzian theory (and imaginary in the Euclidean theory). We recall also that we are interpreting (4.9) and (4.10) as formulas that define a connection $\delta^{Q}$ on the trivial Hilbert space bundle over Teichmuller space $\mathscr{T}$, with fiber $\mathscr{H}=\Gamma\left(\mathscr{M}, \mathscr{L}^{\otimes k}\right)$.

Theorem. The connection $\delta^{Q}$ is projectively flat, with a central curvature that is of type $(1,1)$. It is unitary if $s$ is real.

Concerning the proof of this theorem, the above discussion has already established that the vanishing of the $(2,0)$ and $(0,2)$ parts of the curvature of $\delta^{Q}$ follows from the vanishing of the $(2,0)$ curvature in the case of a compact gauge group. The fact that the $(1,1)$ curvature is central follows by a computation which will be presented at the end of this section. (This computation, which also gives a simple formula for the $(1,1)$ curvature, is rigorous given the formulas of $[21,22]$ for the curvature of the Ray-Singer-Quillen metric on the determinant line bundle of the $\bar{\partial}$ operator; the identities and methods required are exactly those used in [11] for the case of a compact gauge group.) That the connection $\delta^{\mathcal{Q}}$ is unitary for real $s$ is elementary and will be shown in the next subsection.

4.1. Preliminary Properties. Before tackling the question of the $(1,1)$ curvature of the connection $\delta^{Q}$, we will first discuss some properties that can be understood without such an elaborate computation.

Unitarity For Real s. The first issue that we will discuss is unitarity. We first consider the case of real $s$, where a simple answer can be given. Let $($,$) denote$ the hermitian metric on the line bundle $\mathscr{L}^{\otimes k}$ over $\mathscr{M}$, and let $d \mu$ be the measure on $\mathscr{M}$ determined by its symplectic structure (or by its Kähler metric). Then if $s$ is real, or equivalently if $\bar{t}$ is the complex conjugate of $t$, the Hilbert space structure

$$
\langle\chi, \psi\rangle=\int_{\mathscr{M}} d \mu H(\chi, \psi)
$$


is invariant under parallel transport by $\delta^{Q}$. This follows by straightforward computation given the definition of $\delta^{Q}$ in (4.9) and (4.10). One way to express the computation is to note that $\psi$ is invariant under parallel transport by $\delta^{Q}$ if and only if $\widetilde{\psi}=H^{1 / 2} \dot{\psi}$ is invariant under parallel transport by

$$
\tilde{\delta}^{Q}=H^{1 / 2} \delta^{Q} H^{-1 / 2} .
$$

But one straightforwardly computes that

$$
\tilde{\delta}^{Q(1,0)}=\delta^{(1,0)}+\frac{1}{2 t} \nabla_{\underline{\underline{i}}} \delta J^{\underline{i} \underline{\underline{j}} \nabla_{\underline{j}}}-\frac{2}{t} H^{-1 / 2}\left(h \delta^{(1,0)}+\frac{1}{4} \nabla_{\underline{\underline{i}}} \delta J^{\underline{i} \underline{\underline{i}}} \nabla_{\underline{\underline{j}}}\right) H^{1 / 2}
$$

(where in the last term the derivatives are all acting on $H^{1 / 2}$ ) and likewise

$$
\tilde{\delta}^{Q(0,1)}=\delta^{(0,1)}-\frac{1}{2 \bar{t}} \nabla_{\bar{l}} \delta J^{\bar{j}} \nabla_{\bar{j}}+\frac{2}{\bar{t}} H^{-1 / 2}\left(h \delta^{(1,0)}+\frac{1}{4} \nabla_{\bar{l}} \delta J^{\bar{i}} \nabla_{\bar{j}}\right) H^{1 / 2} .
$$

For $s$ real, or in other words if $\bar{t}=k-i$ is the complex conjugate of $t=k+i$, $\widetilde{\delta}^{Q(0,1)}$ is manifestly the adjoint of $\tilde{\delta}^{Q(1,0)}$ with respect to the natural hermitian structure

$$
\langle\tilde{\chi}, \tilde{\psi}\rangle=\int_{\mathscr{M}} d \mu(\tilde{\chi}, \tilde{\psi})
$$

This establishes unitarity for real $s$. Equations (4.14) and (4.15) will incidentally be a useful starting point in Sect. 5 for a study of the special case of a surface $\Sigma$ of genus one.

Unitarity For Imaginary s. We saw in sect. 2.1 that, at least heuristically, one might also expect a unitary structure if $s$ is imaginary. Let us now discuss how this could come about. Let $\mathscr{H}_{s}$ be the quantum Hilbert space for given $s$ (at fixed $k$ ). If $s$ is real, then (4.12) defines a unitary structure on $\mathscr{H}_{s}$. Even if $s$ is not real, then for $\chi \in \mathscr{H}_{\bar{s}}$ and $\psi \in \mathscr{H}_{s}(\bar{s}$ is the complex conjugate of $s),(4.12)$ defines a hermitian pairing

$$
\langle,\rangle: \mathscr{H}_{\bar{s}} \otimes \mathscr{H}_{\mathrm{s}} \rightarrow \mathbb{C},
$$

compatible with parallel transport by $\delta^{Q}$. If in addition we can find a natural isomorphism $\phi: \mathscr{H}_{s} \cong \mathscr{H}_{-s}$, then by defining $\langle\langle\chi, \psi\rangle\rangle=\langle\phi(\chi), \psi\rangle$, we will get a hermitian pairing

$$
\langle\langle,\rangle\rangle: \mathscr{H}_{-\bar{s}} \otimes \mathscr{H}_{s} \rightarrow \mathbb{C}
$$

invariant under parallel transport. For imaginary $s$, one has $-\bar{s}=s$, so in this case $\langle\langle\rangle$,$\rangle would define a hermitian structure on \mathscr{H}_{s}$ which - if positive definite could serve as the sight-for unitary structure.

What could be the origin of the required isomorphism between $\mathscr{H}_{s}$ and $\mathscr{H}_{-s}$ ? In fact, our original Lagrangian (2.1) was invariant under $s \leftrightarrow-s$, combined with $\mathscr{A} \leftrightarrow \overline{\mathscr{A}}$. We have lost this symmetry by using a polarization of the space $\mathscr{W}_{\mathbb{C}}$ of $G_{\mathbb{C}}$ connections which was defined by the equations

$$
0=\frac{\delta}{\delta \mathscr{A}_{z}^{a}} \Psi=\frac{\delta}{\delta \overline{\mathscr{A}}_{\bar{z}}^{a}} \Psi .
$$

Obviously, an equally good gauge invariant real polarization would have been 
defined by the equations

$$
0=\frac{\delta}{\delta \mathscr{A}_{\bar{z}}^{a}} \Psi=\frac{\delta}{\delta \overline{\mathscr{A}}_{\bar{z}}^{a}} \Psi
$$

The underlying symmetry $s \leftrightarrow-s$ is valid if quantization using the polarization (4.19) is equivalent to quantization using (4.20). Since there is no obvious way to interpolate between (4.19) and (4.20) via a connected family of polarizations, this is a more difficult question than the questions that we will address in this paper about the behavior under a continuous variation of the complex structure.

Though we will not arrive at a general answer, our results suggest that the quantizations via (4.19) and (4.20) are in fact equivalent. For example, the formula that we will get presently for the central curvature of $\delta^{Q}$ will be invariant under $s \leftrightarrow-s$; in Sect. 5 it will be possible to see the symmetry under $s \leftrightarrow-s$ very explicitly in genus one.

The considerations just sketeched have an interesting counterpart in the theory of the unitary representations of complex Lie groups. The unitarity of the principal series representations of $G_{\mathbb{R}}$ is analogous to the unitarity of $\delta^{Q}$ for real $s$, while the unitarity of the complementary series representations (which depends on a less obvious choice of hermitian structure) would be analogous to the possible existence of a more exotic unitary structure for imaginary $s$. It is likely that much of the theory of the principal and complementary series has a higher dimensional analog in the Chern-Simons theory.

Left And Right Movers; The Central Charge. One aspect of the relation between $2+1$ dimensional Chern-Simons gauge theory with a compact gauge group and two dimensional current algebra is that if we write $\delta^{Q}=\delta+\mathcal{O}$, then $\mathcal{O}$ corresponds closely to the energy-momentum tensor of the two dimensional theory. The fact that, in the case of compact gauge groups, the $(0,1)$ part of the connection is trivial, $\delta^{Q(0,1)}=\delta^{(0,1)}$, means that the energy-momentum tensor of the two dimensional theory has only a holomorphic contribution.

On the other hand, with the gauge group taken to be complex Lie group, it is clear from (4.9) and (4.10) that if there is a two dimensional theory related to the $2+1$ dimensional $G_{\mathbb{C}}$ theory, then both the holomorphic and antiholomorphic components of the energy momentum tensor are nonzero in this theory. Writing

$$
\delta^{Q(1,0)}=\delta^{(1,0)}+\mathcal{O}^{(1,0)}, \quad \delta^{Q(0,1)}=\delta^{(0,1)}+\mathcal{O}^{(0,1)},
$$

the holomorphic and antiholomorphic components of the energy momentum tensor correspond to $\mathscr{O}^{(1,0)}$ and $\mathcal{O}^{(0,1)}$, respectively. The fact that $\mathcal{O}^{(1,0)}$ and $\mathcal{O}^{(0,1)}$ are both nonzero is a very fundamental difference between the $G_{\mathbb{C}}$ theory and the theory with compact gauge group. In a sense this is the mechanism by which the $G_{\mathbb{C}}$ theory manages to be unitarity despite the noncompactness of the gauge group.

If a $1+1$ dimensional theory related to the $2+1$ dimensional $G_{\mathbb{C}}$ theory does exist, there is a natural conjecture for the values of the left- and right-moving central charges. They should simply be determined by the change of $\mathcal{O}^{(1,0)}$ and $\mathcal{O}^{(0,1)}$ under conformal transformations. As discussed for the compact group case in [11] (see the discussion of Eqs. (2.54) and (4.36) of that paper), this lack of conformal invariance comes from the conformal anomaly in $H$. Under a conformal transformation, $\delta^{(1,0)} \ln H$ and $\delta^{(0,1)} \ln H$ shift by central terms, while the rest of 
$\mathcal{O}^{(1,0)}$ and $\mathcal{O}^{(0,1)}$ is conformally invariant. The left- and right-moving central charges can therefore be read off from the coefficients of $\delta^{(1,0)} \ln H$ and $\delta^{(0,1)} \ln H$ in (4.9) and (4.10), and are

$$
\left(c_{L}, c_{R}\right)=\operatorname{dim} G \cdot\left(1-\frac{2 h}{t}, 1+\frac{2 h}{\bar{t}}\right)
$$

This is the analog of the well known formula

$$
\left(c_{L}, c_{R}\right)=\operatorname{dim} G \cdot\left(\frac{k}{k+h}, 0\right)
$$

in two dimensional current algebra with a compact gauge group.

Let us discuss now the physical implications of (4.22), first for a hypothetical $1+1$ dimensional theory to which the present discussion may be relevant, and then in $2+1$ dimensions.

In $1+1$ dimensions, one wishes $c_{L}$ and $c_{R}$ to be real. Evidently, this is true if and only if $s$ is imaginary. So it is the $2+1$ dimensional Euclidean theory that is most likely to be relevant to a possible $1+1$ dimensional theory. Indeed, the conjectured exotic hermitian structure of the $2+1$ dimensional theory would be used to make modular invariant combinations of conformal blocks in $1+1$ dimensions. The conformal blocks, of course, will not be either holomorphic or antiholomorphic, since the holomorphic and antiholomorphic parts of the stress tensor are both non-zero. We will make these points explicit in Sect. 5.4.

The fact that the central charges depend continuously on a parameter $s$ is unusual in $1+1$ dimensional conformal field theory. The only presently known models with such a phenomenon are Liouville theory and its close cousins. Perhaps the $2+1$ dimensional $G_{\mathbb{C}}$ theories are related to the latter.

Now we move on to consider the implications of the central charge in $2+1$ dimensions. In $2+1$ dimensions (and perhaps also, from some points of view, in $1+1$ dimensions), the key object is the difference

$$
c_{L}-c_{R}=-2 h \operatorname{dim} G \cdot\left(\frac{1}{t}+\frac{1}{\bar{t}}\right) .
$$

This quantity potentially leads $[23,24]$ to a sort of global obstruction to general covariance in three dimensions. When $c_{L}-c_{R}$ does not vanish modulo 24 , the Chern-Simons theory cannot be defined on a "bare" oriented three manifold but requires a choice of framing. Under a change of framing, the amplitudes are multiplied by an integer power of $\exp \left(2 \pi i\left(c_{L}-c_{R}\right) / 24\right)$. From the point of view of gauge theory, one may consider it acceptable to require a framing of the three manifold as part of the definition of the problem. In gravity, however, one might consider it to be a physical principle that the physical amplitudes should be defined for "bare," unframed three manifolds. If that is the right interpretation, then the quantity expressed in (4.24) is a sort of $c$-number anomaly, analogous to the Virasoro central charge, in $2+1$ dimensional general relativity with positive cosmological constant. As in the case of the Virasoro anomaly, one would wish to cancel this term either by choosing $k$ and $s$ judiciously or by coupling to additional matter multiplets. 
The symmetry of (4.24) under $s \leftrightarrow-s$ suggests that the underlying symmetry has indeed not been spoiled by the choice of polarization.

4.2. Analysis of the $(1,1)$ Curvature. In this subsection, we will, finally, show that the $(1,1)$ curvature of the quantum connection $\delta^{Q}$ vanishes except for a central term. This is only difficult calculation in the present paper. Happily, it can be carried out using only the identities that were used in [11] to settle similar questions for the case of a compact gauge group.

Let us recall these identities. First of all, one has the fact that the $(2,0)$ part of the variation of complex structure of $\mathscr{M}$ is holomorphic:

$$
\nabla_{\bar{k}} \delta J^{\underline{i} \underline{j}}=\nabla_{\underline{k}} \delta J^{\bar{i} \bar{j}}=0 .
$$

The other important identities can be deduced from standard considerations about anomalies in two dimensional gauge theory, or, alternatively, from the local families index theorem of $[21,22]$, applied to the determinant of the $\bar{\partial}_{A}$ operator (coupled to the adjoint bundle ad(E)). The relevant anomalies are the anomalies in the two point functions of the currents and stress tensor of a system of two dimensional fermions coupled to the connection $A$. These anomalies are all related to certain statements about the Kähler geometry of the moduli space $\mathscr{M}_{J}$ of stable holomorphic $G_{\mathbb{C}}$ bundles. In formulating these statements (which are equations (4.17-20) in [11]), $R_{\underline{i} \bar{j} \underline{k} \bar{m}}$ will denote the Riemann tensor of $\mathscr{M}_{\mathrm{J}}$, and $R_{\bar{j} \underline{\underline{m}}}=R_{\bar{j} \bar{i} \underline{\underline{i}}}^{\bar{i}}$ will denote its Ricci tensor. The anomaly in the current two point function is equivalent to the statement that

$$
\nabla_{\bar{k}} \nabla_{\underline{m}} \ln H-R_{\bar{k} \underline{m}}=-2 i h \omega_{0 \bar{k} \underline{-}} .
$$

The absence of an anomaly in the off-diagonal two point function connecting the current and the stress tensor is equivalent to the statements that

$$
\begin{aligned}
& \delta^{(1,0)}\left(\nabla_{\bar{m}} \ln H\right)=-\frac{i}{2} \nabla_{\underline{i}} \delta J_{\underline{m}_{\bar{m}}^{i}}^{i} \\
& \delta^{(0,1)}\left(\nabla_{\underline{m}} \ln H\right)=+\frac{i}{2} \nabla_{\bar{l}} \delta J_{\underline{\underline{m}}}^{\bar{i}} .
\end{aligned}
$$

Finally, the anomaly in the two point function of the stress tensor corresponds to the statement that

$$
\delta^{(0,1)} \delta^{(1,0)} \ln H-\frac{1}{4} \delta J^{i}{ }_{j} \delta J^{\bar{j}}{ }_{\underline{i}}=c_{1}\left(\operatorname{Ind}\left(T_{z}\right)\right) .
$$

Here $c_{1}\left(\operatorname{Ind}\left(T_{z}\right)\right)$ is a two form on Teichmüller space (or more precisely, the pullback of such a form to $\mathscr{M} \times \mathscr{T}$ ) which represents the $c$-number central anomaly in two dimensional conformal field theory. We will show that the $(1,1)$ curvature of $\delta^{Q}$ is central by showing that, in fact, it is a multiple of $c_{1}\left(\operatorname{Ind}\left(T_{z}\right)\right)$. The normalization is such that if a projectively flat connection is related to a two dimensional conformal field theory with left- and right-moving central charges $\left(c_{L}, c_{R}\right)$, then the curvature will be

$$
\left(c_{L}-c_{R}\right) \cdot \frac{1}{2 \operatorname{dim} G} \cdot c_{1}\left(\operatorname{Ind}\left(T_{z}\right)\right)
$$

The factor $\operatorname{dim} G$ appears in the denominator here simply because of the way $c_{1}\left(\right.$ Ind $\left.T_{z}\right)$ was defined in [11]. 
In addition to these identities, one should recall some more elementary facts. The quantum Hilbert space is $\mathscr{H}=\Gamma\left(\mathscr{M}, \mathscr{L}^{\otimes k}\right)$, where the line bundle $\mathscr{L}^{\otimes k}$ has a curvature that can be described by the statement

$$
\left[\nabla_{\underline{i}}, \nabla_{\tilde{j}}\right]=-i k \cdot \omega_{0_{i j} \cdot}
$$

Since the $(1,0)$ and $(0,1)$ covariant derivatives are defined by $\nabla_{j}=\frac{1}{2}\left(\delta_{j}{ }_{j}-i J_{j}{ }_{j}\right) \nabla_{i}$, $\nabla_{\bar{j}}=\frac{1}{2}\left(\delta^{i}{ }_{j}+i J^{i}{ }_{j}\right) \nabla_{i}$, one has also

$$
\begin{aligned}
& \delta^{(1,0)}\left(\nabla_{\bar{i}}\right)=+\frac{i}{2} \delta J^{k} \nabla_{\underline{k}}, \\
& \delta^{(0,1)}\left(\nabla_{\underline{i}}\right)=-\frac{i}{2} \delta J_{\underline{\underline{k}}}^{\bar{k}} \nabla_{\bar{k}} .
\end{aligned}
$$

It is convenient to define

$$
\begin{aligned}
& V^{(1,0)}=\nabla_{\underline{i}} \delta J^{i \underline{i} \underline{j}} \nabla_{\underline{j}}+\delta J^{i \underline{j} \underline{j}}\left(\nabla_{\underline{i}} \ln H\right) \nabla_{\dot{j}}+2 k \delta^{(1,0)} \ln H, \\
& V^{(0,1)}=\nabla_{\bar{\imath}} \delta J^{\bar{j} j} \nabla_{\bar{j}}+\delta J^{\bar{j}}\left(\nabla_{\bar{\imath}} \ln H\right) \nabla_{\bar{j}}-2 k \delta^{(0,1)} \ln H .
\end{aligned}
$$

The quantum connection is then

$$
\begin{aligned}
& \delta^{Q(1,0)}=\delta^{(1,0)}+\frac{1}{2 t} V^{(1,0)}+\left(\frac{1}{2}-\frac{k+h}{t}\right) \delta^{(1,0)} \ln H, \\
& \delta^{Q(0,1)}=\delta^{(0,1)}-\frac{1}{2 \bar{t}} V^{(0,1)}+\left(\frac{1}{2}-\frac{k-h}{\bar{t}}\right) \delta^{(0,1)} \ln H .
\end{aligned}
$$

The advantage of writing the connection this way is that one can conveniently borrow some results from the computation in the case of compact gauge group. For instance, the verification of holomorphicity preservation in [11] is equivalent to the statement that

$$
\left[\nabla_{\bar{k}}, V^{(1,0)}\right]=4(k+h)\left[\delta^{(1,0)}, \nabla_{\bar{k}}\right]=2 i(k+h) \delta J_{\bar{k}}^{j} \nabla_{j} .
$$

The mirror image computation with opposite complex structure would give

$$
\left[\nabla_{\underline{k}}, V^{(0,1)}\right]=-4(k-h)\left[\delta^{(0,1)}, \nabla_{\underline{k}}\right]=-2 i(k-h) \delta J^{\bar{j}} \nabla_{\underline{j}} .
$$

The $(1,1)$ part of the curvature is

$$
\begin{aligned}
\left\{\delta^{Q(1,0)}, \delta^{Q(0,1)}\right\}= & -\frac{A}{4 t \bar{t}}+\frac{B}{2 t}-\frac{C}{2 \bar{t}}+\left(\frac{k+h}{t}-\frac{k-h}{\bar{t}}\right) \cdot D+\frac{1}{2 t}\left(\frac{1}{2}-\frac{k-h}{\bar{t}}\right) \cdot E \\
& -\frac{1}{2 \bar{t}}\left(\frac{1}{2}-\frac{k+h}{t}\right) \cdot F
\end{aligned}
$$

with

$$
\begin{aligned}
& A=\left\{V^{(1,0)}, V^{(0,1)}\right\}, \\
& B=\delta^{(0,1)} V^{(1,0)}, \\
& C=\delta^{(1,0)} V^{(0,1)}, \\
& D=\delta^{(1,0)} \delta^{(0,1)} \ln H, \\
& E=\left\{V^{(1,0)}, \delta^{(0,1)} \ln H\right\}, \\
& F=\left\{V^{(0,1)}, \delta^{(1,0)} \ln H\right\} .
\end{aligned}
$$


With the connection $\delta^{Q}$ being given by a second order differential operator, it appears $a$ priori that the $(1,1)$ curvature might be a third order differential operator. However, with the aid of (4.25), such a term is absent. We will now summarize in turn the analysis of possible terms in the $(1,1)$ curvature involving differential operators of second order, first order, or zero ${ }^{\text {th }}$ order.

Second Order Contributions. Second order operators come from $A, B$, and $C$. The contributions can be evaluated using the identities above and are

$$
\begin{aligned}
& A_{2}=-2 i k \delta J^{\bar{m}}{ }_{\underline{i}} \delta J^{\underline{i} \underline{\underline{j}}}\left(\nabla_{\underline{j}} \nabla_{\bar{m}}+\nabla_{\bar{m}} \nabla_{\underline{j}}\right), \\
& B_{2}=-\frac{i}{2} \delta J^{\bar{m}} \delta J^{\underline{i} \underline{j}}\left(\nabla_{\underline{j}} \nabla_{\bar{m}}+\nabla_{\bar{m}} \nabla_{\underline{j}}\right) \text {, } \\
& C_{2}=+\frac{i}{2} \delta J^{\bar{m}}{ }_{\underline{\underline{j}}}^{\underline{\underline{j}}} \delta J^{\underline{\underline{i}} \underline{\underline{j}}}\left(\nabla_{\underline{\underline{j}}} \nabla_{\bar{m}}+\nabla_{\bar{m}} \nabla_{\underline{j}}\right) .
\end{aligned}
$$

Using $t+\bar{t}=2 k$, the total second order contribution cancels in (4.36).

First Order Contributions. With the second order terms being written as in (4.38), the objects $A, B, \ldots, F$ have well defined first order contributions. It is obviously enough to verify that the first order contributions proportional to differential operators of type $(0,1)$ cancel. Contributions of this type come only from $A, B$, and $F$. (It is not obvious that one does not get such a term from $C$, but this is a consequence of the analysis of the $(1,1)$ curvature for compact gauge groups in [11].) One finds

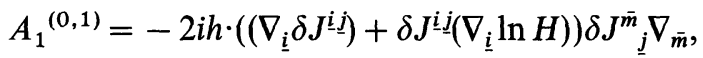

$$
\begin{aligned}
& B_{1}{ }^{(0,1)}=\frac{i}{2} \cdot\left(\left(\nabla_{\underline{i}} \delta J^{\underline{i} \underline{j}}\right)+\delta J^{\underline{\underline{i}} \underline{\underline{j}}}\left(\nabla_{\underline{\underline{i}}} \ln H\right)\right) \delta J^{\bar{m}}{ }_{\underline{\underline{j}}} \nabla_{\bar{m}},
\end{aligned}
$$

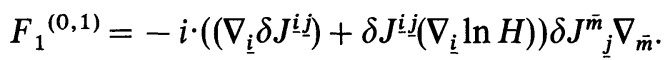

These again cancel when substituted in (4.36).

Zero $^{\text {th }}$ Order Contributions. Finally, we must evaluate the zero ${ }^{\text {th }}$ order contribution to the $(1,1)$ curvature. If we let

$$
\begin{aligned}
& Y=\left(\left(\nabla_{\bar{s}} \delta J^{\bar{s} \bar{\imath}}\right)+\left(\nabla_{\bar{s}} \ln H\right) \delta J^{\bar{s} \bar{\imath}}\right) \cdot\left(\left(\nabla_{\underline{\underline{j}}} \delta J_{\bar{\imath}}^{\underline{j}}\right)+\left(\nabla_{\underline{j}} \ln H\right) \delta J_{\overline{\underline{\imath}}}^{\underline{\underline{i}}}\right) \\
& +\delta J^{\bar{s} \bar{\imath}} \delta J^{\underline{m}} R_{\bar{x}} R_{\bar{s} \underline{i} \bar{i}}^{\bar{x}},
\end{aligned}
$$

then the zero ${ }^{\text {th }}$ order contributions turn out to be

$$
\begin{aligned}
A_{0} & =-i k Y, \\
B_{0} & =-2 k \delta^{(1,0)} \delta^{(0,1)} \ln H, \\
C_{0} & =-2 k \delta^{(1,0)} \delta^{(0,1)} \ln H, \\
D_{0} & =\delta^{(1,0)} \delta^{(0,1)} \ln H, \\
E_{0} & =\frac{i}{2} Y-h \delta J^{j} \underline{\underline{k}}_{\bar{k}} \delta J_{\underline{\underline{j}}}^{\bar{k}}, \\
F_{0} & =-\frac{i}{2} Y+h \delta J_{\overline{\underline{k}}}^{j} \delta J^{\bar{k}} .
\end{aligned}
$$


Inserting these results in (4.36) and using (4.28), we find finally that the $(1,1)$ curvature is central as promised, and in fact we obtain the precise formula

$$
\left\{\delta^{Q(1,0)}, \delta^{Q(0,1)}\right\}=-\left(\frac{h}{t}+\frac{h}{\bar{t}}\right) \cdot c_{1}\left(\operatorname{Ind} T_{z}\right) .
$$

In particular, we recover the resulted stated earlier in Eq. (4.24) that the central charges of a hypothetical $1+1$ dimensional conformal field theory related to the present discussion obey

$$
c_{L}-c_{R}=-2 h \cdot \operatorname{dim} G \cdot\left(\frac{1}{t}+\frac{1}{\bar{t}}\right) .
$$

\section{Genus One}

We will now turn our attention to the special case in which the surface $\Sigma$ is of genus one. As in the analogous situation for a compact gauge group $G[9,11]$, the quantization of the $G_{\mathbb{C}}$ theory can be described in a particularly explicit way for genus one.

Let $T$ be the maximal torus of $G$ and $W$ its Weyl group. Let $T_{\mathbb{C}}$ be the complexification of $T$, which is a complex maximal torus of $G_{\mathbb{C}}$. Because the fundamental group of a genus one surface is abelian, the holonomies of a flat $G$ connection on $\Sigma$ can be simultaneously conjugated into $T$, in a fashion that is unique up to the action of the Weyl group. So the moduli space $\mathscr{M}$ of flat $G$ connections is $T \times T / W$. Since a torus $T \times T$, or its quotient by a finite group, has a standard quantization, there is an obvious guess for how the moduli space of flat $G$ connections should be quantized in genus one. Actually, as explained in $[9,11]$, the correct description, which comes by specializing the genus $g$ story to the case $g=1$, differs from the most obvious guess in some key details, such as a replacement $k \rightarrow k+h$ in some formulas.

Flat $G_{\mathbb{C}}$ connections whose holonomies are semi-simple can similarly be conjugated into $T_{\mathbb{C}} \times T_{\mathbb{C}}$. Thus, if we consider only connections of semi-simple holonomy, it would appear that the moduli space $\mathscr{M}_{\mathbb{C}}$ of flat $G_{\mathbb{C}}$ connections would be $T_{\mathbb{C}} \times T_{\mathbb{C}} / W$. This could be identified with the cotangent bundle $T^{*}(T \times T)$ divided by the Weyl group. (Actually, it can be shown that the effect of the flat connections whose holonomies are not semi-simple is that $\mathscr{M}_{\mathbb{C}}$ for genus one is a smooth manifold obtained by blowing up singularities of $T^{*}(T \times T) / W$.) These facts suggest that the physical Hilbert space for the $G_{\mathbb{C}}$ theory quantized on a genus one surface would be the $W$ invariant subspace of the Hilbert space obtained by quantizing $T^{*}(T \times T)$. It turns out in the $G_{\mathbb{C}}$ case that the naive guess is essentially correct, without any shifts analogous to $k \rightarrow k+h$. This is presumably related to the fact [19] that the canonical line bundle of $\mathscr{M}_{\mathbb{C}}$ is trivial, since the shift $k \rightarrow k+h$ can be interpreted [11,12] as reflecting the role of the canonical line bundle of $\mathscr{M}$.

5.1.1 Use of the Heat Equation. As in the treatment of the compact group case in [11], the key fact that makes a simple treatment of the $G_{\mathbb{C}}$ problem possible in genus one is that the object $H$, the determinant of the Laplacian, obeys a heat 
equation:

$$
\left(h \delta^{(1,0)}+\frac{1}{4} \nabla_{\underline{\underline{i}}} \delta J^{\underline{i} \underline{j}} \nabla_{\underline{j}}\right) H^{1 / 2}=0 .
$$

More precisely, this equation holds if $h$ is defined using flat metrics on $\Sigma$ of unit area. Otherwise, a central term must be added to (5.1).

Equation (5.1) can be considered to be a consequence of the following facts. In genus $g$, the partition function of a system of Majorana fermions in the adjoint representation of $G$ obeys a heat equation because the stress tensor of this system has a Sugawara construction. In genus one, on the other hand, the partition function for such Majorana fermions (coupled to the Dirac operator with the trivial spin structure) is just $H^{1 / 2}$, leading to the heat equation (5.1). This equation is actually closely related to the MacDonald identities and so is studied in the literature on affine Lie algebras.

In any case, once (5.1) is accepted, it is easy to see how to simplify the genus one problem. We saw in Sect. 4.1 that to understand unitarity, it was convenient to conjugate the quantum connection $\delta^{Q}$ by $H^{1 / 2}$ and study the new connection $\tilde{\delta}^{Q}=H^{1 / 2} \delta^{Q} H^{-1 / 2}$, which is given by

$$
\begin{aligned}
& \tilde{\delta}^{Q(1,0)}=\delta^{(1,0)}+\frac{1}{2 t} \nabla_{\underline{i}} \delta J^{\underline{i} \underline{j}} \nabla_{\underline{j}}-\frac{2}{t} H^{-1 / 2}\left(h \delta^{(1,0)}+\frac{1}{4} \nabla_{\underline{\underline{i}}} \delta J^{\underline{i} \underline{j}} \nabla_{\underline{j}}\right) H^{1 / 2}, \\
& \tilde{\delta}^{Q(0,1)}=\delta^{(0,1)}-\frac{1}{2 \bar{t}} \nabla_{\bar{l}} \delta J^{\overline{i j}} \nabla_{\bar{j}}+\frac{2}{\bar{t}} H^{-1 / 2}\left(h \delta^{(0,1)}+\frac{1}{4} \nabla_{\bar{l}} \delta J^{\bar{j} j} \nabla_{\underline{j}}\right) H^{1 / 2}
\end{aligned}
$$

With the aid of (5.1), the conjugated connection reduces to

$$
\begin{aligned}
& \tilde{\delta}^{Q(1,0)}=\delta^{(1,0)}+\frac{1}{2 t} \nabla_{\underline{\underline{\delta}}} \delta J^{\underline{i} \underline{\underline{j}} \nabla_{\underline{j}}}=\delta^{(1,0)}+\frac{1}{4 k}\left(1+\frac{k-i s}{k+i s}\right) \nabla_{\underline{\underline{i}}} \delta J^{\underline{i} \underline{j}} \nabla_{\underline{\underline{j}}}, \\
& \tilde{\delta}^{Q(0,1)}=\delta^{(0,1)}-\frac{1}{2 \bar{t}} \nabla_{\bar{l}} \delta J^{\overline{i j}} \nabla_{\bar{j}}=\delta^{(0,1)}-\frac{1}{4 k}\left(1+\frac{k+i s}{k-i s}\right) \nabla_{\bar{\imath}} \delta J^{\bar{j} j} \nabla_{\underline{\underline{j}}},
\end{aligned}
$$

where we have used $t=k+i s, \bar{t}=k-i$ s. These formulas are even simpler than they may look, because in the genus one case, the intrinsic Kahler geometry of $\mathscr{M}$ is flat, and $\delta J$ is covariantly constant. For a detailed description, see Sect. (5) of [11].

5.2. Finding Explicit Parallel Sections. A further simplification can be obtained in the following way. One introduces the Laplacian $\Delta$ on $\mathscr{M}$, defined by

$$
\Delta=J^{i}{ }_{j} \omega_{0}{ }^{j k} \nabla_{i} \nabla_{k}
$$

Thus,

$$
\left[\delta^{(1,0)}, \Delta\right]=\delta J^{i \underline{k}} \nabla_{\underline{\underline{i}}} \nabla_{\underline{\underline{k}}}, \quad\left[\delta^{(0,1)}, \Delta\right]=\delta J^{\bar{j} j} \nabla_{\bar{\imath}} \nabla_{\bar{j}}
$$

Recalling also that

$$
\left[\nabla_{\underline{i}}, \nabla_{\bar{j}}\right]=-i k \omega_{0 \underline{i} \bar{j}}
$$

(with other components vanishing) and that $J$ acts as multiplication by $+i$ (or $-i$ ) 
on components of type $(1,0)$ (or $(0,1))$ we compute

$$
\begin{aligned}
& {\left[\Delta, \delta J^{\underline{i} \underline{k}} \nabla_{\underline{\underline{i}}} \nabla_{\underline{j}}\right]=4 k \delta J^{\underline{i} \underline{k}} \nabla_{\underline{\underline{i}}} \nabla_{\underline{j}},} \\
& {\left[\Delta, \delta J^{\overline{i k}} \nabla_{\bar{k}} \nabla_{\bar{l}}\right]=-4 k \delta J^{\bar{j}} \nabla_{\bar{\imath}} \nabla_{\bar{j}} .}
\end{aligned}
$$

One can now compute

$$
\begin{aligned}
& e^{-r \Delta} \delta^{(1,0)} e^{r \Delta}=\delta^{(1,0)}+\frac{1}{4 k}\left(1-e^{-4 k r}\right) \delta J^{\underline{i} \underline{j}} \nabla_{\underline{\underline{i}}} \nabla_{\underline{\underline{j}}}, \\
& e^{-r \Delta} \delta^{(0,1)} e^{r \Delta}=\delta^{(0,1)}-\frac{1}{4 k}\left(1-e^{4 k r}\right) \delta J^{\bar{i} \bar{j}} \nabla_{\bar{l}} \nabla_{\bar{j}} .
\end{aligned}
$$

We therefore see that if we pick $r$ so that

$$
e^{-4 k r}=-\frac{k-i s}{k+i s},
$$

then

$$
\tilde{\delta}^{Q}=e^{-r \Delta} \cdot \delta \cdot e^{r \Delta} .
$$

This solves the problem of finding parallel sections of $\tilde{\delta}^{Q}$. As parallel section $\psi$ of $\tilde{\delta}^{Q}$ over the genus one Teichmüller space $\mathscr{T}$ is of the form

$$
\psi=e^{-r \Delta} \psi_{0},
$$

where $\psi_{0}$ is a vector in the fixed Hilbert space $\mathscr{H}=\Gamma\left(\mathscr{M}, \mathscr{L}^{\otimes k}\right)$. The $\mathscr{T}$ dependence in (5.11) arises because the complex structure and Kähler metric of $\mathscr{M}$ and therefore the Laplacian $\Delta$ depend on the choice of a point in $\mathscr{T}$.

At first sight there is a puzzling apparent non-uniqueness of the description (5.11) of explicit parallel sections, since in (5.9), $r$ is determined only modulo an integer multiple of $2 \pi i / 4 k$. It can be seen, however, that (for $k \neq 0$ ) $\Delta$ is of the form

$$
\Delta=2 k N \text {, }
$$

where $N$ is an operator with integer eigenvalues, and that the connection $\delta^{Q}$ commutes with the operator $(-1)^{N}$. Therefore, the indeterminacy in the formula for $r$ essentially factors out of the formula (5.11).

The Case $k=0$. The above formulas are not quite valid for $k=0$, since we have divided by $k$ at various points. In fact, it is easy to see that with $k=0, t=i$, $\bar{t}=-i s,(5.10)$ is still valid, but now with

$$
r=\frac{1}{2 i s} \text {. }
$$

5.3. The Exotic Hermitian Structure. In Sect. 4.1, we showed that in addition to the obvious hermitian pairing $\mathscr{H}_{s} \otimes \mathscr{H}_{s} \rightarrow \mathbb{C}$ which leads to unitarity for real $s$, it is natural to look for an exotic hermitian pairing

$$
\langle\langle,\rangle\rangle: \mathscr{H}_{-\bar{s}} \otimes \mathscr{H}_{s}, \rightarrow \mathbb{C}
$$

compatible with parallel transport by $\tilde{\delta}^{Q}$, which might lead to unitarity for 
imaginary s. We saw that the key to finding such a pairing would be an isomorphism between $\mathscr{H}_{s}$ and $\mathscr{H}_{-s}$.

It is now easy to settle this question in the genus one case. The quantum connections $\tilde{\delta}_{s}^{Q}$ and $\tilde{\delta}^{Q}{ }_{-s}$ (we now make the dependence on $s$ explicit) are conjugate to each other since in fact according to (5.10) they are both conjugate to the trivial connection $\delta$. Therefore, the exotic structure exists. It can easily be written down explicitly. For $\psi \in \mathscr{H}_{s}$ and $\phi \in \mathscr{H}_{-\bar{s}}$, obeying $0=\widetilde{\delta}^{Q}{ }_{s} \psi=\widetilde{\delta}^{Q}{ }_{-\bar{s}} \phi$, or equivalently

$$
0=\delta\left(e^{r \Delta} \psi\right), \quad 0=\delta\left(e^{-\bar{r} \Delta} \phi\right),
$$

we define

$$
\langle\langle\phi, \psi\rangle\rangle=\left\langle\phi, e^{2 r \Delta} \psi\right\rangle
$$

This is equivalent to

$$
\langle\langle\phi, \psi\rangle\rangle=\left\langle e^{-\bar{r} \Delta} \phi, e^{r \Delta} \psi\right\rangle,
$$

which makes it obvious that $\delta\langle\langle\phi, \psi\rangle\rangle=0$.

For imaginary $s$, one has $-\bar{s}=s$, so (5.16) is in fact a new hermitian structure on $\mathscr{H}_{s}$. Is it positive definite?

For $k=0,(5.13)$ shows that $r$ is real if $s$ is imaginary, so

$$
e^{2 r \Delta}=\left(\frac{i s-k}{i s+k}\right)^{N},
$$

where $N$ has non-negatve integer eigenvalues. Here $u=(i s-k) /(i s+k)$ is real for imaginary $s$. The operator in (5.18) is bounded above by one for $|u|<1$. For $s$ such that $1>u>0$, this operator is positive definite, so (5.16) defines a unitary structure. Actually, even for $0>u>-1$, it would be possible to achieve positivity in (5.16) by multiplying by hand by a factor of $(-1)^{N}$, since this operator commutes with $\delta$, $\tilde{\delta}^{Q}$, etc. One might suspect, however, that this last step (which is possible because the moduli spaces in genus one are quotients of linear spaces by discrete groups) is unlikely to generalize to genus $\neq 1$.

In the theory of the unitary representations of $G_{\mathbb{C}}$, the exotic hermitian structure exists for all $k$ but is positive only for $k=0$. In the gauge theory problem, at least for genus one, this structure is positive (in a suitable range of $s$ ) for all $k$.

Looking back to the relation (4.13) between the original quantum connection $\delta^{Q}$ and the modified connection $\tilde{\delta}^{Q}$ which preserves the unitary structure (5.16), we see that in terms of wave functions $\Phi=H^{-1 / 2} \phi, \Psi=H^{-1 / 2} \psi$ invariant under parallel transport by the original connection, the exotic unitary structure is

$$
\langle\langle\Phi, \Psi\rangle\rangle=\left\langle\Phi, H^{1 / 2} e^{2 r \Delta} H^{1 / 2} \Psi\right\rangle .
$$

5.4. Attempt at a $1+1$ Dimensional Interpretation. We will now make a tentative attempt at a $1+1$ dimensional interpretation of the above results; in fact, we will propose a candidate for the partition function of a $1+1$ dimensional theory that may be related to the $2+1$ dimensional discussion.

In the case of a compact gauge group $G$, the relation between conformal blocks in $1+1$ dimensions and quantum states in $2+1$ dimensions can be described as follows. In $2+1$ dimensions, a quantum state is a wave function $\Psi(A ; \tau)(A$ denotes a connection and $\tau$ denotes a point in Teichmüller space) which is annihilated by 
the quantum connection $\delta^{Q}$. The corresponding conformal block is obtained simply by setting $A=0$ and is $\Psi(\tau)=\Psi(0 ; \tau)$. The modular invariant partition function of $1+1$ dimensional current algebra (corresponding to the diagonal modular invariant combination of conformal blocks) can be described as follows. Picking a basis of orthonormal quantum states $\Psi_{\alpha}(A ; \tau)$ (orthonormal, that is, with respect to the unitary structure preserved by $\delta^{Q}$ ), the partition function of the $1+1$ dimensional theory is

$$
Z(\tau)=\sum_{\alpha}\left|\Psi_{\alpha}(0 ; \tau)\right|^{2}
$$

with $\left|\Psi_{\alpha}(A ; \tau)\right|^{2}$ being the norm of $\psi_{\alpha}(A ; \tau)$ with respect to the metric on the line bundle $\mathscr{L}^{\otimes k}$ that is being quantized.

Imitating these steps, we can try to propose a candidate for the partition function of a $1+1$ dimensional theory that is related to the $G_{\mathbb{C}}$ theory in $2+1$ dimensions. One still has quantum states $\Psi_{\alpha}(A ; \tau)$ which are annihilated by an appropriate quantum connection $\delta^{Q}$; setting $A=0$, one gets candidates $\Psi_{\alpha}(\tau)=\Psi_{\alpha}(0 ; \tau)$ for the conformal blocks in $1+1$ dimensions. One even has the explicit description (5.11) of these conformal blocks. A significant difference from the case of a compact group is that in the $G_{\mathbb{C}}$ case, these candidate conformal blocks are neither holomorphic nor anti-holomorphic in $\tau$ (since the $(1,0)$ and $(0,1)$ parts of the quantum connection $\delta^{Q}$ are both non-trivial).

The next step is to form a modular invariant combination of conformal blocks. New issues arise, because the sum in (5.20) will be an infinite sum in the case of the $G_{\mathbb{C}}$ theory. It is the imaginary $s$ theory, with its exotic unitary structure defined in (5.19), that seems to be more suitable. The analog of (5.20) can be worked out formally and is

$$
Z(\tau)=\left\langle A=0\left|H^{-1 / 2} e^{+2 r \Delta} H^{-1 / 2}\right| A=0\right\rangle,
$$

which is equivalent to

$$
Z(\tau)=\frac{1}{H(A=0 ; \tau)}\left\langle A=0\left|e^{+2 r \Delta}\right| A=0\right\rangle .
$$

This formula raises several questions. First, $H$ is defined as the determinant of the Laplacian on the underlying Riemann surface $\Sigma$, and this has zero modes for $A=0$. However, it is fairly natural in (5.22) to interpret $H$ as the determinant of the Laplacian in the space orthogonal to the zero modes. A more serious puzzle is, perhaps, that the matrix element $\left\langle A=0\left|e^{+2 r \Delta}\right| A=0\right\rangle$ makes sense only for negative $r$. Though there is no contradiction here, and negative $r$ does indeed arise in a suitable range of imaginary $s$, this requirement is perplexing since it is positive $r$ that one wants in the $2+1$ dimensional theory.

5.5. Analog of the Knizhnik-Zamolodchikov Equation. Canonical quantization of Chern-Simons gauge theory with a compact gauge group, for a Riemann surface $\Sigma$ of genus $g$ with $n$ marked points (the marked points being labeled with additional data such as representations of the gauge group), is naturally described by giving a projectively flat connection on the quantum bundle over Teichmüller space $\mathscr{T}_{g, n}$. The connection can be described in terms of differential equations. For the case of $g=0$, the appropriate equations are particularly simple and were first obtained 
by Knizhnik and Zamolodchikov [25] from the point of view of $1+1$ dimensional current algebra. On the complex $z$ plane (actually the Riemann sphere, including the point at infinity), we consider configurations of $n$ points $z_{1}, \ldots, z_{n}$, with associated representations $R_{1}, \ldots, R_{n}$. The quantum wave function $\Psi\left(z_{1}, \ldots, z_{n}\right)$ (which has values in $R_{1} \otimes \cdots \otimes R_{n}$; this is not indicated explicitly) is required to obey the Knizhnik-Zamolodchikov equation:

$$
\begin{aligned}
\left(\frac{\partial}{\partial z_{i}}-\frac{1}{k+h} \sum_{j \neq i} \frac{T_{(i)} \cdot T_{(j)}}{z_{i}-z_{j}}\right) \Psi & =0 \\
\frac{\partial}{\partial \bar{z}_{i}} \Psi & =0 .
\end{aligned}
$$

Here $T_{(i)}$ are the generators of the symmetry group $G$ for the $i^{\text {th }}$ particle, and $T_{(i)} \cdot T_{(j)}=\sum_{a} T_{(i)}{ }^{a} T_{(j)}{ }^{a}$ is an invariant bilinear expression in these generators. The second equation reflects the fact that in $1+1$ dimensional current algebra with compact group, the $(0,2)$ part of the stress tensor vanishes, so the correlation functions are holomorphic. This second equation is often left implicit. The equations in (5.23) are compatible since the operators on the left commute; this is the flatness of the connection.

For the case of a complex gauge group $G_{\mathbb{C}}$, we have in this paper treated in considerable detail the case of a Riemann surface of genus $g$ without marked points. It would be highly desirable to extend the analysis to the general case of genus $g$ with $n$ marked points. Such an extension would greatly clarify the whole question of whether there is a $1+1$ dimensional quantum field theory related to the $2+1$ dimensional $G_{\mathbb{C}}$ theory, and would also probably make it possible to compute many more physical properties in $2+1$ dimensional gravity with positive cosmological constant (and to understand the three manifold invariants and knot invariants associated with the $G_{\mathbb{C}}$ theory). Such an extension is beyond the scope of the present paper. However, it is easy to guess what should be the analog of the Knizhnik-Zamolodchikov equation in genus zero, and we will now do this.

With each marked point $z_{i}$, we associate a unitary representation $R_{i}$ of $G_{\mathbb{C}}$. If $s$ is real, we take the $R_{i}$ to be principal series representations of $G_{\mathbb{C}}$, and if $s$ is imaginary, we take them to be complementary series representations.

Now, $G_{\mathbb{C}}$ is a Lie group whose complex dimension is $\operatorname{dim} G$ and whose real dimension is $2 \cdot \operatorname{dim} G$. One can take a real basis of the $G_{\mathbb{C}}$ Lie algebra in which the generators are $t_{a}, s_{a}$, with $t_{a}$ being the generators of a compact subgroup and so obeying $\left[t_{a}, t_{b}\right]=f_{a b}{ }^{c} t_{c}$ (with some structure constants $f$ ), and the $s_{b}$ transforming in the adjoint representation of the compact subgroup and obeying $\left[t_{a}, s_{b}\right]=f_{a b}{ }^{c} s_{c}$, and $\left[s_{a}, s_{b}\right]=-f_{a b}{ }^{c} t_{c}$. If one now defines $t_{a}{ }^{ \pm}=\frac{1}{2}\left(t_{a} \pm i s_{a}\right)$, one finds $\left[t_{a}{ }^{ \pm}, t_{b}{ }^{ \pm}\right]=$ $f_{a b}{ }^{c} c_{c}{ }^{ \pm}$, while $\left[t_{a}{ }^{+}, t_{b}{ }^{-}\right]=0$. This corresponds to the fact that if one regards the $G_{\mathbb{C}}$ Lie algebra as a real Lie algebra and complexifies it again, one gets the Lie algebra of $G_{\mathbb{C}} \times G_{\mathbb{C}}$. (For the case in which $G_{\mathbb{C}}=S L(2, \mathbb{C})$, regarded as the Lorentz group in $3+1$ dimensions, this manipulation is often used in order to study the representations of the Lorentz group.)

In the spirit of the analysis we have made in this paper for genus $g$ without marked points, the obvious analog of the Knizhnik-Zamolodchikov equation is 
now

$$
\begin{aligned}
& \left(\frac{\partial}{\partial z_{i}}-\frac{2}{t} \sum_{j \neq i} \frac{t_{(i)}^{+} \cdot t_{(j)}^{+}}{z_{i}-z_{j}}\right) \Psi=0, \\
& \left(\frac{\partial}{\partial \bar{z}_{i}}+\frac{2}{\bar{t}} \sum_{j \neq i} \frac{t_{(i)}{ }^{-} \cdot t_{(j)}}{\bar{z}_{i}-\bar{z}_{j}}\right) \Psi=0 .
\end{aligned}
$$

This equation would determine the conformal blocks in the $n$ point functions of a hypothetical $1+1$ dimensional theory related to our discussion. These blocks, of course, are neither holomorphic nor anti-holomorphic, but, after understanding the unitary structure, one could attempt to combine them into physical correlation functions. The monodromies of the differential equations (5.24) would determine the $G_{\mathbb{C}}$ analogs of the Jones representations of the braid group.

\section{Additional Properties}

In this section we will briefly consider certain additional questions.

In Sect. 6.1, we consider the gravitational interpretation of the $S L(2, \mathbb{C})$ theory. In Sects 6.2 and 6.3, we consider the extent to which some familiar structures of the theory with compact gauge group generalize to the $G_{\mathbb{C}}$ case. The structures in question are "duality" of conformal blocks, and the role of unitary representations of the loop group.

6.1. Gravitational Interpretation. The arguments [4] claiming to show that $2+1$ dimensional general relativity can be reinterpreted as a gauge theory involve combining the vierbein $e$ and the spin connection $\omega$ into a gauge field $\mathscr{A}=\omega_{a} J^{a}+e_{a} P^{a}$, and then claiming that diffeomorphisms of $(e, \omega)$ are equivalent to gauge transformations of $\mathscr{A}$. This claim is valid if it can be assumed that the vierbein is invertible. Once one formulates the theory as a gauge theory, however, one drops the requirement that the vierbein should be invertible. The consequences of this step must be understood in deciding whether the gauge theory really has a gravitational interpretation.

In the canonical formulation, in which one is assigning a physical Hilbert space $\mathscr{H}_{\Sigma}$ (of "solutions of the Wheeler-de Witt equation" or its analog in a different polarization) to a Riemann surface $\Sigma$, this question can be posed rather sharply. (In a more general situation in which one is considering topology-changing amplitudes, the correct physical principles are not entirely clear; we will not attempt to explore these matters here.) In quantum gravity, $\mathscr{H}_{\Sigma}$ should be constructed by quantizing the moduli space $\mathscr{Z}_{\boldsymbol{A}}$ of solutions of the Einstein equations with cosmological constant $\Lambda$. In the gauge theory interpretation, one is instead quantizing a moduli space $\mathscr{M}_{\boldsymbol{A}}$ of flat connections on $\Sigma$ (with gauge group $S L(2, \mathbb{R}) \times S L(2, \mathbb{R}), I S O(2,1)$, or $S L(2, \mathbb{C})$ for $\Lambda$ negative, zero, or positive). (In the cases of $S L(2, \mathbb{R}) \times S L(2, \mathbb{R})$ and $I S O(2,1)$, one considers flat bundles of the right Euler class, as was explained in [4].) The equivalence of quantum gravity with gauge theory holds in the canonical formalism if it is true that $\mathscr{M}_{\Lambda}=\mathscr{Z}_{\Lambda}$. In fact, there is always a natural map $\phi: \mathscr{Z}_{\Lambda} \rightarrow \mathscr{M}_{\Lambda}$, since every solution of the Einstein equations does determine a flat connection. The question arises of whether $\phi$ is 
invertible. This will be so if every flat connection representing a point in $\mathscr{M}_{\Lambda}$ can by a gauge transformation be put in a form in which the vierbein is invertible and if there is only a unique way to do this, up to a diffeomorphism and local Lorentz transformation.

For $\Lambda=0$ and $\Sigma$ of genus $\geqq 2, \mathrm{G}$. Mess has recently proved that this is so [18]. Actually, for $\Lambda=0$, Mess' work exhibits a very interesting feature, namely that every classical solution has a past singularity or a future singularity, but not both. (This is easily seen to be true for solutions whose holonomy lies in an $S O(2,1)$ subgroup of the gauge group [4].) It is intriguing to ask whether this corresponds to a possibility of building CPT violation into quantum gravity. Mess also argues (but does not prove) that the desired relation $\mathscr{Z}_{\Lambda}=\mathscr{M}_{\Lambda}$ holds for $\Lambda<0$. (In this case he also argues that every classical solution has both a past singularity and a future singularity, something that can easily be seen for the solutions whose holonomy lies in an $S O(2,1)$ subgroup.)

However, for $\Lambda>0$, the situation is more subtle. (For $\Lambda>0, \mathscr{M}_{\Lambda}$ is what we have previously been calling $\mathscr{M}_{\mathbb{C}}$.) Mess shows that every projective structure on $\Sigma$ (a projective structure is a covering of $\Sigma$ by open sets $\mathcal{O}_{i}$, with maps $u_{i}: \mathcal{O}_{i} \rightarrow \mathrm{CP}^{1}$, such that where they are defined, the $u_{i} u_{j}^{-1}$ are in $S L(2, \mathbb{C})$ ) gives rise to a gravitational solution with positive cosmological constant (in fact, to two of them, one with a past singularity and one with a future singularity). He conjectures that in fact for positve $\Lambda, \mathscr{Z}_{\Lambda}$ is the space of such prejective structures. If so, then it can be shown that almost every flat $S L(2, \mathbb{C})$ connection (the exceptions are a set of very high codimension) can by a gauge transformation be put in a form in which the vierbein is invertible. Let us throw away the exceptions (which are, for instance, the flat connections with holonomy contained in $S U(2)$ ) and denote the space of flat connections which can be gauged to a form with an invertible vierbein as $\mathscr{M}^{*}$. The key subtlety is now that every flat connection in $\mathscr{M}^{*}{ }_{\Lambda}$ can be gauged to a form with invertible vierbein in infinitely many different ways (which are not equivalent by diffeomorphisms and local Lorentz transformations), so the map $\phi$ from $\mathscr{Z}_{\Lambda}$ to $\mathscr{M}^{*}{ }_{\Lambda}$ is infinitely-many-to-one for $\Lambda>0$. The one redeeming feature of the situation is that the map $\phi$ is locally an isomorphism, so that locally in phase space the mapping between gravity and gauge theory is valid (according to Mess's conjectures) also for $\Lambda>0$.

The implications of this for quantum gravity would appear to be that the $S L(2, \mathbb{C})$ Hilbert spaces $\mathscr{H}$ that we have constructed in this paper would be only subspaces of the actual gravitational Hilbert spaces $\mathscr{H}_{\text {grav }}$. Roughly speaking, the choice of a point in $\mathscr{Z}_{\Lambda}$ corresponding to a given point in $\mathscr{M}^{*}{ }_{\Lambda}$ would give a new quantum number in the gravitational problem, which we have omitted in our gauge theory treatment.

Here is a somewhat more precise account. From the standpoint of geometric quantization [13-16], quantization arises by defining a prequantum line bundle $\mathscr{L}_{\text {pr }}$ over $\mathscr{M}^{*}{ }_{A}$, introducing a polarization, and then defining $\mathscr{H}^{*}$ to consist of sections of $\mathscr{L}_{\mathrm{pr}}$ that are covariantly constant along the leaves of the polarization. ${ }^{6}$ (With this definition, the Hilbert space $\mathscr{H}$ obtained by quantizing $\mathscr{M}_{\Lambda}$ may be a

\footnotetext{
6 What follows is the one point in this paper in which we use the point of view of geometric quantization in a substantive way. Our arguments until this point have been based on standard field theoretic considerations and are therefore more clearly valid than the comments that follow
} 
proper subspace of the Hilbert space $\mathscr{H}^{*}$ obtained by quantizing $\mathscr{M}^{*}{ }_{\Lambda}$.) Given a map $\phi: \mathscr{Z} \rightarrow \mathscr{M}^{*}$ that is locally an isomorphism, one can pull back the prequantum line bundle and the polarization from $\mathscr{M}^{*}$ to $\mathscr{Z}$ and define $\mathscr{H}_{\text {grav }}$, the quantization of $\mathscr{Z}$, to consist of sections of the pullback of $\mathscr{L}_{\mathrm{pr}}$ that are covariantly constant along the leaves of the pulled-back polarization. With this definition, the Hilbert space $\mathscr{H}$ that we have obtained in this paper by quantizing the $S L(2, \mathbb{C})$ moduli space $\mathscr{M}_{\Lambda}$ is naturally a subspace of $\mathscr{H}_{\text {grav }}$ (since any element of $\Gamma\left(\mathscr{M}_{\phi} \mathscr{L}_{\text {pr }}\right.$ ) can be restricted to $\mathscr{M}^{*}{ }_{\Lambda}$ and then pulled back to $\mathscr{Z}$ ).

In actuality, quantization of $\mathscr{M}_{\Lambda}$ is carried out by considering a family of polarizations of $\mathscr{M}_{\Lambda}$ and constructing a flat connection $\delta^{Q}$ over the parameter space $\mathscr{T}$ of this family. There is no difficulty in principle in carrying out this step for the enlarged gravitational Hilbert space $\mathscr{H}_{\text {grav }}$, since the differential equation that was used to define $\delta^{Q}$ can be restricted to $\mathscr{M}^{*}{ }_{\Lambda}$ and then pulled back from $\mathscr{M}^{*}{ }_{\Lambda} \times \mathscr{T}$ to $\mathscr{Z}_{\Lambda} \times \mathscr{T}$. Since the inclusion of $\mathscr{H}$ in $\mathscr{H}_{\text {grav }}$ commutes with parallel transport by $\delta^{Q}$, local observables like the central charge are unmodified by replacing $\mathscr{H}$ with $\mathscr{H}_{\text {grav }}$.

One reason to believe that it is really $\mathscr{H}_{\text {grav }}$ that is the right Hilbert space for the gravitational theory is that, since the space of projective structures is a deformation of the cotangent bundle of Teichmüller space, the Hilbert space $\mathscr{H}_{\text {grav }}$ goes over for $\Lambda \rightarrow 0$ to the Hilbert space (of square integrable functions on Teichmüller space) which seems natural [4] for quantum gravity with $\Lambda=0$. It is nevertheless dissatisfying to make an $a d$ hoc replacement of $\mathscr{H}$ by the larger Hilbert space $\mathscr{H}_{\text {grav }}$ sketched above, which does not at the moment have a natural field theoretic construction. Perhaps there is a new physical principle, presently unknown, of generally covariant quantum field theory, that singles out the subspace $\mathscr{H}$ as the "correct" one, even though it is $\mathscr{H}_{\text {grav }}$ that corresponds more closely to the canonical quantization of gravity. In this case, however, it is not clear what the new principle would lead to for $\Lambda \leqq 0$.

6.2. Duality. One of the crucial properties of rational conformal field theory $[26,27]$ is "duality." Among other things, duality means that for every way of describing a Riemann surface $\Sigma$ as a thickening of a trivalent graph $\Gamma$, one gets an explicit basis for the Hilbert space $\mathscr{H}_{\Sigma}$ of conformal blocks of that theory. In the case of current algebra of a compact symmetry group $G$, the explicit basis is obtained by labeling the lines in $\Gamma$ by certain representations of $G$ and the vertices by certain couplings of representations $[27,28]$. It seems that such a description holds in the case of gauge group $S L(2, \mathbb{R})[23,8]$. The question arises of whether a similar description holds in the $G_{\mathbb{C}}$ case. We will now indicate very briefly that it does.

We have seen that in the $G_{\mathbb{C}}$ theory, the Hilbert space associated to a surface $\Sigma$ has a description as $\Gamma\left(\mathscr{M}, \mathscr{L}^{\otimes k}\right)$, where $\mathscr{M}$ is the moduli space of representations of the fundamental group of $\Sigma$ into the compact group $G$. This may be compared to the case of "holomorphic orbifolds" [29], where one considers a finite group $F$, introduces the set $\mathscr{S}$ of representations of the fundamental group of $\Sigma$ in $F$, and (in the untwisted case) defines a Hilbert space consisting of functions on $\mathscr{S}$. The problem of holomorphic orbifolds is clearly analogous to the $G_{\mathbb{C}}$ gauge theory, with the following dictionary: $F \leftrightarrow G ; \mathscr{S} \leftrightarrow \mathscr{M}$; functions on $\mathscr{S} \leftrightarrow L^{2}$ functions on $\mathscr{M}$; twisting of the holomorphic orbifold (derived [30] from an element of 
$\left.H^{4}(B F, \mathbb{Z})\right) \leftrightarrow$ replacement of functions on $\mathscr{M}$ by sections of $\mathscr{L}^{\otimes k}$ with $k \neq 0$. We may therefore borrow the reasoning of [29], where it was shown how duality comes about in the case of holomorphic orbifolds. Indeed, there it was shown that the space of conformal blocks in the theory of holomorphic orbifolds has a "dual" description in which the lines in a graph $\Gamma$ (of which a Riemann surface $\Sigma$ is a thickening) are labeled by giving an element of $F$ (up to conjugacy) and a representation of its centralizer. The same is therefore true in the $G_{\mathbb{C}}$ theory, with $F$ replaced by $G$.

Under a duality similar to that of E. Verlinde [27], a conjugacy class of $G$ together with a representation of its centralizer is more or less the data corresponding to a unitary principal series representation of $G_{\mathbb{C}}$, so it is probably possible to interpret this by saying that the lines in $\Gamma$ are labeled by unitary representations of $G_{\mathbb{C}}$.

There is not a continuously variable parameter analogous to $s=t-\bar{t}$ in the holomorphic orbifold theory; this theory gives representations of the mapping class group similar to those that appear for $G_{\mathbb{C}}$ in the limit $s \rightarrow \infty$ where the quantum connection simplifies.

6.3. Unitary Representations of the Loop Group of $G_{\mathbb{C}} \cdot 2+1$ dimensional Chern-Simons gauge theory with a compact gauge group $G$ is related to $1+1$ dimensional current algebra, with symmetry group $G$, and thereby to unitary highest weight representations of (a central extension of) the loop group $L G$. It is natural to wonder whether the $2+1$ dimensional $G_{\mathbb{C}}$ theory is similarly related to some unitary representations of $L G_{\mathbb{C}}$

The relevant unitary representations of $L G$ arise by quantizing the homogeneous symplectic manifold $L G / G$ (here we are dividing $L G$ on the right by the subgroup of the loop group consisting of constant maps to $G$ ). Similarly, one might expect to get unitary representations of $L G_{\mathbb{C}}$ by quantizing $L G_{\mathbb{C}} / G_{\mathbb{C}}$ in a suitable sense.

In $2+1$ dimensional Chern-Simons theory with gauge group $G$, the problem of quantizing $L G / G$ naturally arises as follows [1]. One quantizes the $2+1$ dimensional theory on a three manifold of the form $D \times \mathbb{R}^{1}$, where $D$ is a disc and $\mathbb{R}^{1}$ represents time. The Chern-Simons Lagrangian is invariant under the group $\hat{G}_{1}$ of gauge transformations that are the identity on the boundary of $D \times \mathbb{R}^{1}$, which is $L \times \mathbb{R}^{1}, L$ being a loop. One quantizes the space of $G$ connections on $D$ by picking a complex polarization, just as one would do if the boundary of $D$ were empty. The classical phase space of flat $G$ connections on $D$ modulo $\widehat{G}_{1}$ is $L G / G$. Quantizing $L G / G$ like any other moduli space of flat $G$ connections, one gets back the usual unitary highest weight representations of $L G$, realized as $H^{0}\left(L G / G, \mathscr{L}^{\otimes k}\right)$, with $\mathscr{L}$ the basic holomorphic line bundle over $L G / G$.

Possible unitary representations of $L G_{\mathbb{C}}$ related to the $G_{\mathbb{C}}$ Chern-Simons theory should be found in a similar way. One formulates the $G_{\mathbb{C}}$ theory on $D \times \mathbb{R}^{1}$. The space of $G_{\mathbb{C}}$ connections on $D$ can be quantized by picking a real polarization, just as we have done in this paper in the case that the boundary of $D$ is empty. The classical phase space of flat $G_{\mathbb{C}}$ connections on $D$ modulo $\widehat{G}_{\mathbb{C}, 1}$ is $L G_{\mathbb{C}} / G_{\mathbb{C}}$. Quantizing $L G_{\mathbb{C}} / G_{\mathbb{C}}$ by the methods that we have used in this paper, one will at least formally get unitary representations of (a central extension of) $L G_{\mathbb{C}}$. With the sort of real polarization that we have been using, $L G_{\mathbb{C}} / G_{\mathbb{C}}$ will have an 
identification (depending on a choice of complex structure on $D$ ) with the cotangent bundle $T^{*}(L G / G)$, and thus the Hilbert space on which $L G_{\mathbb{C}}$ is realized will be more or less the space of $L^{2}$ sections $\Gamma\left(L G / G, \mathscr{L}^{\otimes k}\right)$.

Let diff $S^{1}$ be the group of diffeomorphisms of a circle. In conformal field theory in general, the-Hilbert space attached to a circle has a non-trivial action of two copies of (central extensions of) diff $S^{1}$ - one for left-movers and one for right-movers. In the case of representations of $L G$, the action of one of the two copies of $\operatorname{diff} S^{1}$ is trivial, essentially because in quantizing the Chern-Simons theory with compact gauge group, the quantum connection $\delta^{Q}$ has a $(0,1)$ piece which is trivial. In the unitary representations of $L G_{\mathbb{C}}$ constructed as above, both copies of diff $S^{1}$ will act nontrivially, essentially because the $G_{\mathbb{C}}$ theory has a quantum connection $\delta^{Q}$ with non-trivial $(1,0)$ and $(0,1)$ pieces. These two copies of diff $S^{1}$ will not intertwine with $L G_{\mathbb{C}}$ in the elementary way that is familiar from the $L G$ case.

Attempted At A Concrete Description. Let us attempt to give a concrete description of some representations of $L G_{\mathbb{C}}$ with the right properties (though I will not try to deduce the following description directly from the above discussion). ${ }^{7}$

First we recall the situation for representations of $L G$. One considers the Lie algebra

$$
\left[J^{a}(\sigma), J^{b}\left(\sigma^{\prime}\right)\right]=i f^{a b}{ }_{c} J^{c}(\sigma) \delta\left(\sigma-\sigma^{\prime}\right)+\frac{i k \delta^{a b}}{4 \pi} \delta^{\prime}\left(\sigma-\sigma^{\prime}\right) .
$$

Here $\sigma$ and $\sigma^{\prime}$, which range from 0 to $2 \pi$, are points on a circle $S$; $k$ is a positive integer; and the $J^{a}(\sigma)$, with $a=1 \cdots \operatorname{dim} G$, are hermitian "currents." Expanding in modes, $J^{a}(\sigma)=\sum_{n} e^{-i n \sigma} J_{n}{ }^{a}$, one notes that the operators $J_{0}{ }^{a}$ generate a finite dimensional Lie algebra isomorphic to the Lie algebra $\mathbf{g}$ of $G$. Picking an irreducible representation $R$ of $\mathbf{g}$ in states $\left|\lambda_{r}\right\rangle, r=1 \cdots p$ and postulating that

$$
J_{n}{ }^{a}\left|\lambda_{r}\right\rangle=0, \quad n>0
$$

determines an irreducible highest weight representation of $L G$. Further study shows that this representation is unitary if $R$ obeys certain restrictions.

Now, we consider the case of $L G_{\mathbb{C}}$. The complexified current algebra can be written in the form

$$
\begin{aligned}
& {\left[\mathscr{J}^{a}(\sigma), \mathscr{J}^{b}\left(\sigma^{\prime}\right)\right]=i f^{a b}{ }_{c} \mathscr{J}^{c}(\sigma) \delta\left(\sigma-\sigma^{\prime}\right)+\frac{i t \delta^{a b}}{8 \pi} \delta^{\prime}\left(\sigma-\sigma^{\prime}\right),} \\
& {\left[\overline{\mathscr{J}}^{a}(\sigma), \overline{\mathscr{J}}^{b}\left(\sigma^{\prime}\right)\right]=i f^{a b} \overline{\mathscr{J}}^{c}(\sigma) \delta\left(\sigma-\sigma^{\prime}\right)+\frac{i \bar{t} \delta^{a b}}{8 \pi} \delta^{\prime}\left(\sigma-\sigma^{\prime}\right),} \\
& {\left[\mathscr{J}^{a}(\sigma), \overline{\mathscr{J}}^{b}\left(\sigma^{\prime}\right)\right]=0 .}
\end{aligned}
$$

We suppose $t=k+i$ s, $\bar{t}=k-i$, with $k \in \mathbb{Z}$ and $s$ real. Of course, $\overline{\mathscr{J}}$ is the hermitian

\footnotetext{
${ }^{7}$ It is interesting to compare the following to some unitary representations of $\operatorname{LSL}(2, \mathbb{R})$ that were described in $[31]$ and may conceivably play a role in $S L(2, \mathbb{R})$ Chern-Simons theory in three dimensions
} 
adjoint of $\mathscr{J}$. Expanding in Fourier modes, with $\mathscr{J}^{a}=\sum_{n} e^{-i n \sigma} \mathscr{J}_{n}{ }^{a}$, the $\mathscr{J}_{0}{ }^{a}$ generate a copy of the complex Lie algebra $\mathbf{g}_{\mathbb{C}}$ of $G_{\mathbb{C}}$. Picking a unitary representation $R_{\mathbb{C}}$ of this Lie algebra in states $\left|\psi_{r}\right\rangle$, we now postulate the analog of (6.2), namely

$$
\begin{array}{ll}
\mathscr{J}_{n}{ }^{a}\left|\psi_{r}\right\rangle=0, & n>0 \\
\overline{\mathscr{J}}_{n}{ }^{a}\left|\psi_{r}\right\rangle=0, & n<0 .
\end{array}
$$

Hopefully, these formulas if suitably interpreted determine an irreducible, unitary representation of $L G_{\mathbb{C}}$. The main difficulty in interpreting them is that, because of the use of a real polarization, the states $\left|\psi_{r}\right\rangle$ are not normalizable in the unitary structure that one should attempt to define, but are similar to plane waves in quantum mechanics.

Acknowledgements. I am indebted to Michael Atiyah, Nigel Hitchin, Graeme Segal, Steve Carlip, Geoffrey Mess, Scott Axelrod, and Steve DellaPietra for many discussions.

\section{References}

1. Witten, E.: Quantum field theory and the Jones polynomial. Commun. Math. Phys. 121, 351 (1989); Gauge theories and integrable lattice models, Nucl. Phys. B322, 351 (1989); Gauge theories, vertex models, and quantum groups. Nucl. Phys. B330, 285 (1990)

2. Achúcarro, A., Townsend: A Chern-Simons actions for three dimensional anti-De Sitter supergravity theories. Phys. Lett. 180B, 89 (1986)

3. Rocek, M., van Nieuwenhuizen, P.: Class. Quantum Grav. 3, 43 (1986)

4. Witten, E.: $2+1$ dimensional gravity as an exactly soluble system. Nucl. Phys. B311, 46(1988)

5. Witten, E.: Topology-Changing amplitudes in $2+1$ dimensional gravity. Nucl. Phys. B323, 113 (1989)

6. Carlip, S.: Exact quantum scattering in $2+1$ dimensional gravity. Nucl. Phys. B324, 106 (1989)

7. Knizhnik, V. G., Polyakov, A. M., Zamolodchikov, A. B.: Fractal structure of $2 d$ gravity. Mod. Phys. Lett. A 3, 319 (1988)

8. Verlinde, H.: Conformal field theory, 2-D quantum gravity, and quantization of Teichmüller space, Princeton preprint PUPT-89/1140

9. Elitzur, S., Moore, G., Schwimmer, A., Seiberg, N.: Remarks on the canonical quantization of the Chern-Simons-Witten theory. IAS preprint HEP-89/20

10. Bos, M., Nair, V.: Coherent state quantization of Chern-Simons theory. Columbia University preprint (May, 1989)

11. Axelrod, S., DellaPietra, S., Witten, E.: Geometric quantization of Chern-Simons gauge theory, to appear in Jour. Diff. Geom. (May, 1991).

12. Hitchin, N.: Flat connections and geometric quantization. Commun. Math. Phys. 131, 347-380 (1990)

13. Kostant, B.: Orbits, Symplectic Structures, and Representation Theory. Proc. of the U.S.-Japan Seminar in Differential Geometry, Kyoto, Japan, 1965. On Certain Unitary Representations Which Arise From a Quantization Theory. Lecture Notes in Math., Vol 6, Battelle Seattle Rencontres, Berlin, Heidelberg, New York: Springer 1970, p. 237. Orbits And Quantization Theory. Proc. Int. Congress of Mathematicians, Nice, 1970, p. 395. Quantization and Unitary Representations. Lecture Notes in Math. Vol 170, Berlin, Heidelberg, New York: Springer 1970, p. 87. Line Bundles and the Prequantized Schrodinger Equation. Coll. Group Theoretical Methods in Physics, Centre de Physique Theorique, (Marseille, 1972) p. 81. Symplectic Spinors. Symposia Mathematica (Rome), Vol XIV (1974), p. 139. On the Definition of Quantization. Geometric Symplectique et Physique Mathématique, Coll. CNRS, No. 237 (Paris, 1975), p. 187. Quantization and Representation Theory. Proc. Oxford Conference on Group Theory and Physics, (Oxford, 1977); Kostant, B., Auslander, L.: Polarization and unitary representations of solvable Lie groups, Invent. Math. 753 (1971) 
14. Souriau, J.-M.: Quantification geometrique. Commun. Math. Phys. 1, 374 (1966), Structures Des Systems Dynamiques, Paris: Dunod 1970

15. Sniatycki, J.: Geometric quantization and quantum mechanics, Berlin, Heidelberg, New York: Springer 1980

16. Woodhouse, N.: Geometric quantization. Oxford: Oxford University Press 1980

17. Carlip, S., de Alwis, S. P.: Wormholes in $2+1$ dimensions. IAS preprint HEP-89/52 (1989)

18. Geoffrey Mess,: Flat Lorentz spacetimes. Preprint (to appear)

19. Hitchin, N.: The self-duality equations on a Riemann surface. Proc. London Math. Soc. 3, 55,59 (1987)

20. Ray, D., Singer, I. M.: R-Torsion and the Laplacian on Riemannian Manifolds. Adv. Math. 7, 145 (1971). Analytic Torsion of Complex Manifolds. Ann. Math. 98, 154 (1973)

21. Quillen, D.: Determinants of Cauchy-Riemann operators over a Riemann surface. Funct. Anal. Appl. 19, 31 (1985)

22. Bismut, J., Freed, D.: The analysis of elliptic families. Commun. Math. Phys. 106, 159 (1986)

23. Witten, E.: The central charge in three dimensions. In Physics and Mathematics of Strings, Brink, L., Friedan, D., Polyakov, A. M. (eds.) Singapore: World Scientific 1990

24. Atiyah, M. F.: On framings of 3-manifolds. Oxford University preprint (1989), to appear in Topology.

25. Knizhnik, V. G., Zamolodchikov, A. B.: Current algebra and Wess-Zumino model in two dimensions. Nucl. Phys. B247, 83 (1984)

26. Belavin, A., Polyakov, A., Zamolodchikov, A.: Infinite conformal symmetry in twodimensional quantum field theory. Nucl. Phys. B241, 333 (1984)

27. Verlinde, E.: Fusion rules and modular transformations in $2 d$ conformal field theory. Nucl. Phys. B300, 360 (1988)

28. Moore, G., Seiberg, N.: Polynomial equations for rational conformal field theories. Phys. Lett. B212, 360 (1988) Classical and quantum conformal field theory. Nucl. Phys. B.

29. Dijkgraaf, R., Vafa, C., Verlinde, E., Verlinde, H.: Operator algebra of orbifold models. Commun. Math. Phys. 123, 485 (1989)

30. Dijkgraaf, R., Witten, E.: Topological gauge theories and group cohomology. Commun. Math. Phys. 129, 393 (1990)

31. Jakobsen, H. P., Kac, V. G.: A new class of unitarizable highest weight representations of infinite dimensional Lie algebras, preprint

Communicated by S.-T. Yau 